\title{
Searching for Dark Matter at Hadron Colliders
}

\author{
Andrew Askew, ${ }^{1}$ Sushil Chauhan, ${ }^{2}$ Björn Penning, ${ }^{3}$ William Shepherd, ${ }^{4}$ and Mani Tripathi ${ }^{2}$ \\ ${ }^{1}$ Department of Physics, Florida State University, Tallahassee, FL, USA \\ ${ }^{2}$ Department of Physics, University of California Davis, Davis CA, USA \\ ${ }^{3}$ Fermilab National Laboratory, Batavia IL, \\ USA and The University of Chicago, Chicago, IL, USA \\ ${ }_{4}^{4}$ Santa Cruz Institute for Particle Physics and Department of Physics, \\ University of California Santa Cruz, Santa Cruz, CA, USA
}

(Dated: September 5, 2018)

\begin{abstract}
Theoretical and experimental techniques employed in dedicated searches for dark matter at hadron colliders are reviewed. Bounds from the 7 and $8 \mathrm{TeV}$ proton-proton collisions at the LHC on dark matter interactions have been collected and the results interpreted. We review the current status of the Effective Field Theory picture of dark matter interactions with the Standard Model. Currently, LHC experiments have stronger bounds on operators leading to spin-dependent scattering than direct detection experiments, while direct detection probes are more constraining for spin-independent scattering for WIMP masses above a few $\mathrm{GeV}$.

PACS numbers: 95.35.+d,13.85.Qk, 13.85.Rm

Keywords: Dark matter, LHC, WIMP
\end{abstract}




\section{INTRODUCTION}

With the discovery of the Higgs boson at the LHC [1, 2] the problem of electroweak symmetry breaking has been solved, albeit superficially. Significant theoretical reasons remain for the involvement of additional physics in the electroweak sector, chief among them the sensitivity of the Higgs mass to any new higher scale introduced to our theory. However, the experimental demand for new particles to regulate weak boson scattering, pending confirmation of the Higgs properties, appears to have been resolved. While there remains an expectation that there should be new particles at the TeV scale, the Standard Model (SM) continues to describe all the measurements made at colliders throughout history surprisingly well.

The next great experimental requirement for new physics is due to an entirely different sort of measurement. Particle physics has historically been driven by looking at interactions on ever smaller length scales, where new interactions were required in order to understand the behavior observed at slightly longer distances. The great new arrival on the scene for particle physics is the overwhelming evidence for the existence of dark matter (DM). Various evidences and inferences which lead to the widely accepted existence of this fundamentally new component of the universe have been laid out in great detail in [3].

The only constraints that can be placed on dark matter from cosmological concerns are that it have the correct cosmological energy density, that it be massive so that it can act (at least for gravitational purposes) as pressureless matter, and that it not interact so strongly as to either disturb the well-understood cosmic microwave background [4, 5] or to fail to collapse sufficiently to explain the observed large-scale structure of the universe [5]. While these requirements are fairly straightforward to state, much effort has been invested in understanding their precise implications for the particle physics of dark matter.

Many different models and mechanisms for the properties and production of dark matter exist in the early universe, with the masses predicted for dark matter ranging from the $\mu \mathrm{eV}$ scale to beyond the Planck scale. Each of these different models has distinct motivations and assumptions for the physics of the early universe.

For some time the most popular models of DM have been based on the so-called Weakly Interacting Massive Particle (WIMP) Miracle [6]. The basic observation is that if DM is in thermal equilibrium in the early universe and leaves equilibrium only when its interactions 
become rare compared to the Hubble rate then the mass and annihilation cross section of DM are enough to determine the remaining 'thermal relic' abundance of DM in the universe. The miracle is that, by simply assuming that DM is some stable particle which is involved in electroweak symmetry breaking, one finds the correct order of magnitude for the DM abundance. Since neutral, stable particles are fairly common in models introduced to explain other theoretical problems, notably the gauge hierarchy problem, this has been considered to be a very promising mechanism for DM production.

Guided by this consensus that WIMP DM candidates are particularly well-motivated, a robust experimental effort is underway to either discover DM, or constrain candidate theories. It is widely agreed that, in order to develop a reasonably complete understanding of the physics of DM, multiple different detection and discovery techniques are required. The two main avenues which have concentrated on the DM specifically are generally termed direct and indirect detection, depending on whether the search is for WIMPs themselves or their annihilation products.

Direct detection involves searching for WIMPs scattering off of nuclei of ordinary matter. These scatterings impart small amounts of kinetic energy (typically $<100 \mathrm{keV}$ ) to the recoiling nuclei, thus making it necessary to build detectors capable of low energy detection thresholds. Such experiments are deployed in laboratories with a large overburden of earth, and are instrumented within elaborate shields against cosmic ray interactions in order to remain effectively background-free. For direct searches, the energy threshold, target mass and exposure time become the limiting factors governing the sensitivity of the experiments. This scattering rate can be related by the particle physics model of DM to the annihilation cross section, which in turn must have the appropriate behavior to explain the DM energy density of the universe.

Indirect detection, by contrast, involves searches for the products of the very annihilation processes that are responsible for establishing the DM relic density. These processes should still be going on today, especially in regions where the local DM densities are much higher than they are in our galactic neighborhood. The unfortunate disadvantage associated with indirect detection is the background induced by 'ordinary' astrophysics. For example, If one were to consider only the signal strength from DM annihilations, by far the most promising target for indirect detection would be the galactic center, but there is a relatively unconstrained astrophysical background to any signals that could be produced there. Searches 
in the Galactic Center have indeed found new contributions which are consistent with a DM annihilation origin [7-12], but the signals are also compatible with astrophysical backgrounds and/or statistical fluctuations. Dwarf spheroidal galaxies, which are satellites of the Milky Way, are very rich in DM content and provide ideal sources for indirect searches, however, all attempts at measuring an excess of gamma rays in those systems has yielded null results [13].

Complementary to these two approaches, collider searches [14] have historically focused on the other aspects of the particle theories that happened to contain DM candidates, searching for their strongly-coupled new particles and using the DM candidate as a convenient tag for new physics events in the form of missing energy. Recently, a more direct focus on DM at colliders was proposed [15] and rapidly adopted [16 39] by the collider community. The goal is to relate the pair production rate of DM at colliders to the annihilation and scattering rates at more traditional DM-oriented experiments, while making as few assumptions about other, possibly related new physics as possible. This approach is made possible in large part by applying effective field theory techniques to the problem of DM interactions.

Collider searches for DM have their own advantages and weaknesses. Both direct and indirect detection signals lose strength as the DM candidate becomes lighter due to the smaller amount of energy available in each interaction. In contrast, colliders are able to copiously produce light particles, and hence do not suffer from threshold effects in their search for low-mass DM. However, they suffer from parton distribution function suppression for high DM masses (above hundreds of $\mathrm{GeV}$ ), where the other two search techniques are more robust, even though the rates are lowered due to the reduction in DM number density. The greater uncertainty associated with DM searches at colliders, however, is whether or not a putative signal is actually caused by the true DM in the universe or an imposter particle that is stable only on collider timescales, but not cosmological ones. This is not at all a concern in other techniques, as they are looking for the currently extant DM particles, rather than producing a new pair of particles.

In this article, we begin by reviewing the effective field theory and other theoretical tools which have been employed in designing and interpreting collider searches for dark matter (section II). We proceed to outline the general experimental approaches employed in searching for direct dark matter production (section III), followed by a discussion of the current leading results derived from those searches at the LHC and elsewhere (section IV). 


\section{MODEL INDEPENDENT DARK MATTER INTERACTIONS}

When trying to understand DM interactions with ordinary matter, it can be useful to 'de-focus' one's theoretical eye a bit, and consider generic interactions rather than specific models of DM. This, hopefully, helps us understand that which is truly generic to DM physics as opposed to the specific predictions of one particular model. A fully general approach, assuming nothing about DM except that it exists, has some mass, and is (at least phenomenologically) stable, is achieved by working in an effective field theory framework and writing down interactions between the DM and SM fields of interest. An exhaustive approach to interactions of DM with hadronic matter was undertaken in [40]. Here we will choose a smaller but representative set of possible interactions to consider. We will consider scalar and fermionic DM particles, assuming always that the DM is completely uncharged under the SM gauge group, and look only at those interactions that can induce appreciable signals at tree level in direct detection experiments. Note that, while in principle it is possible for scattering off of leptons to contribute to at least some direct detection searches, these contributions are strongly suppressed compared to nuclear scatterings by the kinematics involved. To avoid possible confusion, we will refer to fermionic DM candidates uniformly as $\chi$ and scalar DM candidates as $\phi$.

In the spirit of healthy scientific agnosticism, it must be acknowledged that even these most basic assumptions about the physics of DM are subject to argumentation. Many models exist where DM is charged under the SM weak interactions. In fact, these models are the original WIMP models, where the DM explicitly interacted with the weak force. Other models consider DM that is milicharged under electromagnetism [41]. In general, the model-independent technique strives to understand the physics of all models as much as is possible, however, there will always be exceptions to its applicability.

The representative interactions which we will consider are listed in table $\mathbb{1}$. Note that they have names which originated in [40] and can be considered purely historical. Each operator is preceded by an assumed Wilson coefficient, and we have chosen those which are most standard in the literature.

The scalar-type operators D1 and C1 are of a higher dimensionality than they naïvely appear, with an additional suppression of $m_{q} / \Lambda$. This factor is chosen for two reasons. First, the scalar-type operators violate $S U(2)_{L}$, and thus technically must also couple to the Higgs 
(a) Operators for Dirac fermion DM

\begin{tabular}{|l|c|c|c|}
\hline Name & Operator & Dimension & SI/SD \\
\hline D1 & $\frac{m_{q}}{\Lambda^{3}} \bar{\chi} \chi \bar{q} q$ & 7 & SI \\
D5 & $\frac{1}{\Lambda^{2}} \bar{\chi} \gamma^{\mu} \chi \bar{q} \gamma_{\mu} q$ & 6 & SI \\
D8 & $\frac{1}{\Lambda^{2}} \bar{\chi} \gamma^{\mu} \gamma^{5} \chi \bar{q} \gamma_{\mu} \gamma^{5} q$ & 6 & SD \\
D9 & $\frac{1}{\Lambda^{2}} \bar{\chi} \sigma^{\mu \nu} \chi \bar{q} \sigma_{\mu \nu} q$ & 6 & SD \\
D11 & $\frac{\alpha_{s}}{\Lambda^{3}} \chi G^{\mu \nu} G_{\mu \nu}$ & 7 & SI \\
\hline
\end{tabular}

(b)Operators for Complex scalar DM

\begin{tabular}{|l|c|c|c|}
\hline Name & Operator & Dimension & SI/SD \\
\hline C1 & $\frac{m_{q}}{\Lambda^{2}} \phi^{\dagger} \phi \bar{q} q$ & 6 & SI \\
C3 & $\frac{1}{\Lambda^{2}} \phi^{\dagger} \overleftrightarrow{\partial}_{\mu} \phi \bar{q} \gamma^{\mu} q$ & 6 & SI \\
C5 & $\frac{\alpha_{s}}{\Lambda^{2}} \phi^{\dagger} \phi G^{\mu \nu} G_{\mu \nu}$ & 6 & SI \\
\hline
\end{tabular}

TABLE I: Lowest-dimensional operators which couple singlet DM candidates to hadronic matter and give unsuppressed contributions to direct detection scattering of DM off of a nucleus. The fourth column indicates whether the primary direct detection signal due to that operator is spinindependent (SI) or spin-dependent (SD). As scalars have no spin, all the listed operators for scalar DM give rise only to SI direct detection signals.

boson to be gauge invariant. Replacing the Higgs by its vacuum expectation value gives a form similar to the one we have chosen. The choice to scale the operators by each quark's mass rather than by a uniform factor of $v / \Lambda$ is motivated by the conjecture of Minimal Flavor Violation (MFV) [42], which protects new models from being strongly constrained by flavor physics observables by insisting that all flavor violation be proportional to the SM Yukawa matrices. It is worth noting that the normalization of D9 actually isn't very well-motivated from a theoretical point of view. In principle, the same suppression by $m_{q}$ that is present for the scalar-type operators, D1 and C1, should also be present there, since the operator violates $S U(2)_{L}$ in the same way. This normalization for the tensor operator is standard, however, because this is the normalization which is most naturally probed in direct detection experiments. We have listed only the operators whose contributions to direct detection scattering are not suppressed by the small dark matter velocity. It is worth noting, however, that other operators are possible and have bounds similar to those derived for these operators from collider searches, while they are effectively unbounded by direct detection due to the suppression of the scattering cross section.

Operators of this type, in some combination, suffice to describe all the interactions of DM with hadronic matter, provided that the new particles involved in the interactions (of an extended theory) are much heavier than the scales at which we are probing the interactions 
between the two. This is manifestly the case in direct detection, where the probe energy is a tiny fraction of the DM mass due to the low velocity of DM in the galactic halo. However, it is much more suspect for a collider search, where the dark matter pair is generally produced relativistically and thus can have kinetic energy comparable to its mass. Nonetheless, these conditions can be met in many models, and these operators provide a good understanding of the physics of those scenarios.

One critical feature of these interactions which is often overlooked is their mixing. In particular, D1 and C1, which are very weakly constrained at the LHC due to the explicit quark mass suppression in the operator, can induce (at one-loop level) D11 and C5, respectively. The diagrams which cause this mixing are completely analogous with those responsible for the gluon fusion production of the Higgs boson [43]. The latter operators are very strongly constrained by colliders, which highlights the importance of tracking these effects. This was originally pointed out in [44, wherein the effects of the full one-loop production are also calculated, outside of the heavy-quark limit in which one can truly treat the operators as mixing with no new form factors. They find that, while the bounds from the gluon-coupling operators cannot be directly transferred onto the scalar-type operators, they do not overstate the bounds by more than about a factor of 20 in cross-section (which is only a factor of 1.6 or 2.1 in the suppression scale of D11 or C5, respectively), still giving much stronger results than the scalar operator alone at tree-level.

Multiple approaches to understanding the range of applicability of these operators have been developed. One can require that the coupling between the particle needed to induce the interaction and the particles under consideration must be small enough to be perturbative as a first step, as is done in [40]. More sophisticated approaches to understanding the range of applicability of these theories utilize the requirement of unitarity [45], which is generally a weaker but more robust constraint than the former, or have taken the approach of considering explicit models with relatively light interaction-mediating particles [46 50], a technique which has since been adopted by the CMS collaboration as well [51]

The "light mediator" models are really examples of simplified models as recommended in [52], where it is assumed that only one new particle (beyond the DM candidate) describes accurately all of the interactions of DM with ordinary matter. Generally, each of the operators in Table I is replaced by a propagating particle, either scalar or vector, which has appropriate couplings to the DM candidate and to hadronic matter. The operator can again 
be inserted in the limit where the mediator mass becomes very large. As a simple example, the Lagrangian for a simplified model completion of operator D1 would be

$$
\mathcal{L} \supset m_{\Phi}^{2} \Phi^{\dagger} \Phi+g_{\chi} \Phi \bar{\chi} \chi+g_{q} \frac{m_{q}}{m_{\Phi}} \Phi \bar{q} q+\text { h.c. }
$$

where $\Phi$ is the new mediator particle and the factor $\frac{m_{q}}{m_{\Phi}}$ is just a normalization introduced into the quark coupling to maintain the MFV conjecture value for the interaction. It is straightforward to see that in the limit of large $m_{\Phi}$ this gives back the operator D1, with the assignment $\Lambda^{3} \equiv \frac{m_{\Phi}^{3}}{g_{q} g_{\chi}}$. One of the chief uncertainties in a model of this type is due to the width of the mediator particle. The standard assumption that is made is that the width scales linearly with the mass of the mediator, as one would expect on naïve dimensional grounds. However, it was shown in [53] that the choice of behavior for the width has important implications for the strength and behavior of the bounds. We also note in passing that the narrow-width approximation is generically used for these particles, and in some cases has been extrapolated beyond its realm of applicability. In particular, conclusions have been reached that the bounds from a model with a very heavy mediator are actually weaker than those from the effective operator due to effects of the mediator width, but these are misleading, as the width of a far off-shell particle is not identical to the width of the same particle on-shell, and needs to be recomputed at the appropriate invariant mass.

Finally, it is important to note that even these simplified models can be overly simple from the point of view of DM physics. As explored in [54], the dark matter's various interactions with SM particles are very commonly dictated by interactions with non-hadronic particles. In fact, it is often some mixture of different particles which is important, with the dominant species changing depending on the particular type of process we are considering. For example, a 'vanilla' SUSY neutralino with the correct relic density generated by the welltempering mechanism [55] generically annihilates preferentially into weak vector bosons, but scatters in direct detection primarily through the Higgs boson. All of the approaches above, even if extended to consider interactions with either gauge or Higgs bosons, as done by [56, 57], have implicitly assumed that, for all the processes we are interested in, the DM interacts dominantly with the same SM field. Thus we emphasize again the importance of verifying the assumptions made in the model-independent results we quote below when trying to apply them to a given more-complete model of DM physics. Ultimately, when a complete model is under consideration, fully focused theoretical vision is best. 


\section{COLLIDER SEARCHES FOR DARK MATTER}

Common to all DM searches is the signature of missing transverse momentum (often called missing transverse energy, $\mathbb{E}_{\mathrm{T}}$ ) caused by the WIMPs escaping the detector. As described

in Sec. II these events can be produced in association with Standard Model particles, most notably photons and jets (either from quarks or gluons) but also $W, Z$ or even Higgs bosons and heavy quarks ( $b$ - and top-quark). These particles produced in association with the WIMP pair will recoil against the invisible particles. The common signature is therefore a large value of $\mathbb{E}_{\mathrm{T}}$ and a back-to-back topology between $\mathrm{E}_{\mathrm{T}}$ and the Standard Model particle used for tagging. In the ATLAS and CMS experiments analyses using jets, photons and $W / Z$ bosons have been performed with more final states yet to be analyzed. In the following we will briefly describe the existing analyses ordered according to their signature. In all analyses only data which has passed the relevant quality criteria of ATLAS and CMS have been used in the searches reviewed.

The direct detection experimental results from LUX [58], SIMPLE [59], SuperCDMS [60], IceCube [61] (assuming for concreteness a $W^{+} W^{-}$dominant annihilation channel), PICASSO 62, and XENON-100 [63], which represent the best current bounds for WIMPnucleon scattering, are also compared to get a complete overview of the current status of DM searches, including limits on WIMP-nucleon cross section and scale of these interactions $(\Lambda)$.

\section{A. Mono-photon searches}

ATLAS and CMS performed searches in events containing one photon and large missing transverse energy. In this final state the dominant background process is $Z(\rightarrow \nu \nu)+\gamma$ production with smaller backgrounds coming from $W / Z+\gamma$ and $W / Z+$ jet production in which electrons or jets are mis-identified as photons.

The ATLAS collaboration has performed a mono-photon analysis using $4.7 \mathrm{fb}^{-1}$ of $p p$ collisions at a center of mass energy of $\sqrt{s}=7 \mathrm{TeV}$ [64]. Events are required to pass an $\mathbb{E}_{\mathrm{T}}$ trigger with a threshold of $\mathrm{E}_{\mathrm{T}}>70 \mathrm{GeV}$. Events are selected if they contain a photon candidate with $p_{T}(\gamma)>150 \mathrm{GeV}$ within a pseudo-rapidity of $|\eta|<2.37$, excluding the transition region between barrel and endcaps of the ATLAS detector $(1.35<|\eta|<1.52)$. 
These events are then required to contain $\mathbb{E}_{\mathrm{T}}>150 \mathrm{GeV}$ and not more than than one jet with $p_{T}$ (jet) $>30 \mathrm{GeV}$ and $|\eta|<4.5$ are. Finally photons, jets and $\mathrm{E}_{\mathrm{T}}$ have to be well separated by $\Delta \phi>0.4$ between $\mathbb{E}_{\mathrm{T}}$ and either photon or jet, and $\Delta R>0.4$ between photon and jet.

Backgrounds are modeled using data and cross-checked in control regions. Multijet background production is estimated using data control regions. The smaller background processes of single-top, diboson, $\gamma \gamma, \gamma+$ jet production are modeled using Monte Carlo simulations. Figure 1 shows the $\mathbb{E}_{\mathrm{T}}$ after full signal selection. No significant excess over the Standard Model expectation is observed.

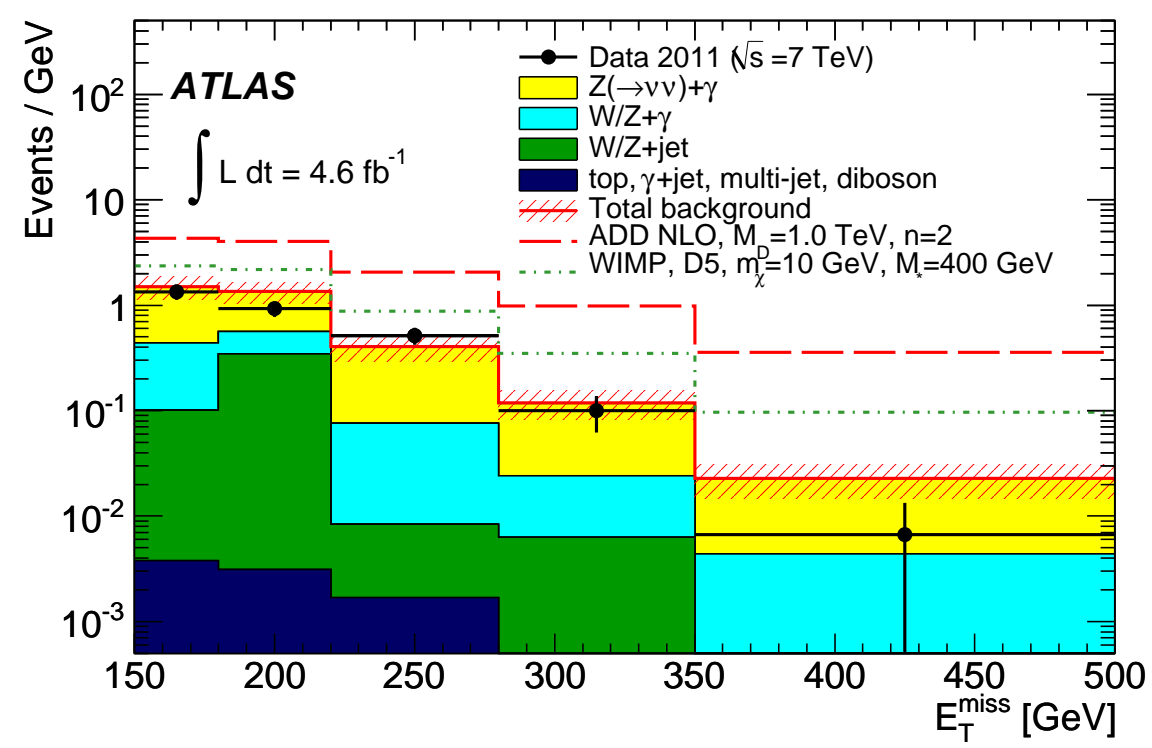

FIG. 1: $\mathbb{E}_{\mathrm{T}}$ distribution observed after full event selection in the ATLAS mono-photon search [64.

The CMS collaboration also performed a mono-photon analysis using $5.0 \mathrm{fb}^{-1}$ of $p p$ collisions at a center of mass energy of $\sqrt{s}=7 \mathrm{TeV}$ [65]. The analysis uses single photon triggers which are fully efficient for the selected signal. The photons are required to fulfill $p_{T}(\gamma)>145 \mathrm{GeV}$ and to be within the central electromagnetic calorimeter $|\eta|<1$.44. The missing transverse energy of these events has to exceed $\mathbb{E}_{\mathrm{T}}>130 \mathrm{GeV}$. Events with a muon present or significant hadronic activity, signified by either having a track with $p_{T}>20 \mathrm{GeV}$ or a jet with $p_{T}($ jet $)>40 \mathrm{GeV}$ within $|\eta|<3.0$ and $\Delta R>0.5$ of the photon axis, are rejected.

The CMS collaboration has recently update this search [66] with $19.6 \mathrm{fb}^{-1}$ of data collected at $\sqrt{s}=8 \mathrm{TeV}$. The analysis is similar except few differences: events are require to 
have $p_{T}(\gamma)>145 \mathrm{GeV}$ and $\mathrm{E}_{\mathrm{T}}>140 \mathrm{GeV}$, jet veto is optimized with one jet is allowed to be present in the event below $p_{T}($ jet $)<30 \mathrm{GeV}$. These jets are counted only if they qualify the pile-up jet identification criteria and have $p_{T}$ (jet) $>20 \mathrm{GeV}$ within $|\eta|<3.0$. The lepton veto is used where events are rejected if a lepton is present in the event with $p_{T}^{l}>10 \mathrm{GeV}$ and also satisfies the stringent lepton identification requirements. The SM prediction with all these selections is also tested using a control region where lepton veto are reversed to select a phase space dominated by SM electroweak backgrounds.

In both version of this search, instrumental backgrounds from electrons or jets reconstructed as photons are estimated using data in control regions. Additional "out of time backgrounds" from cosmic muons and beam halo are estimated using timing requirements, and the remaining backgrounds are estimated using simulation. Figure 2 shows the corresponding $p_{T}(\gamma)$ spectrum from $\sqrt{s}=7 \mathrm{TeV}$ and $\sqrt{s}=8 \mathrm{TeV}$ searches. The $\sqrt{s}=7 \mathrm{TeV}$ analysis estimate a cut-and-count based limits while in $8 \mathrm{TeV}$ analysis shapes are exploited to get the cross section limits.
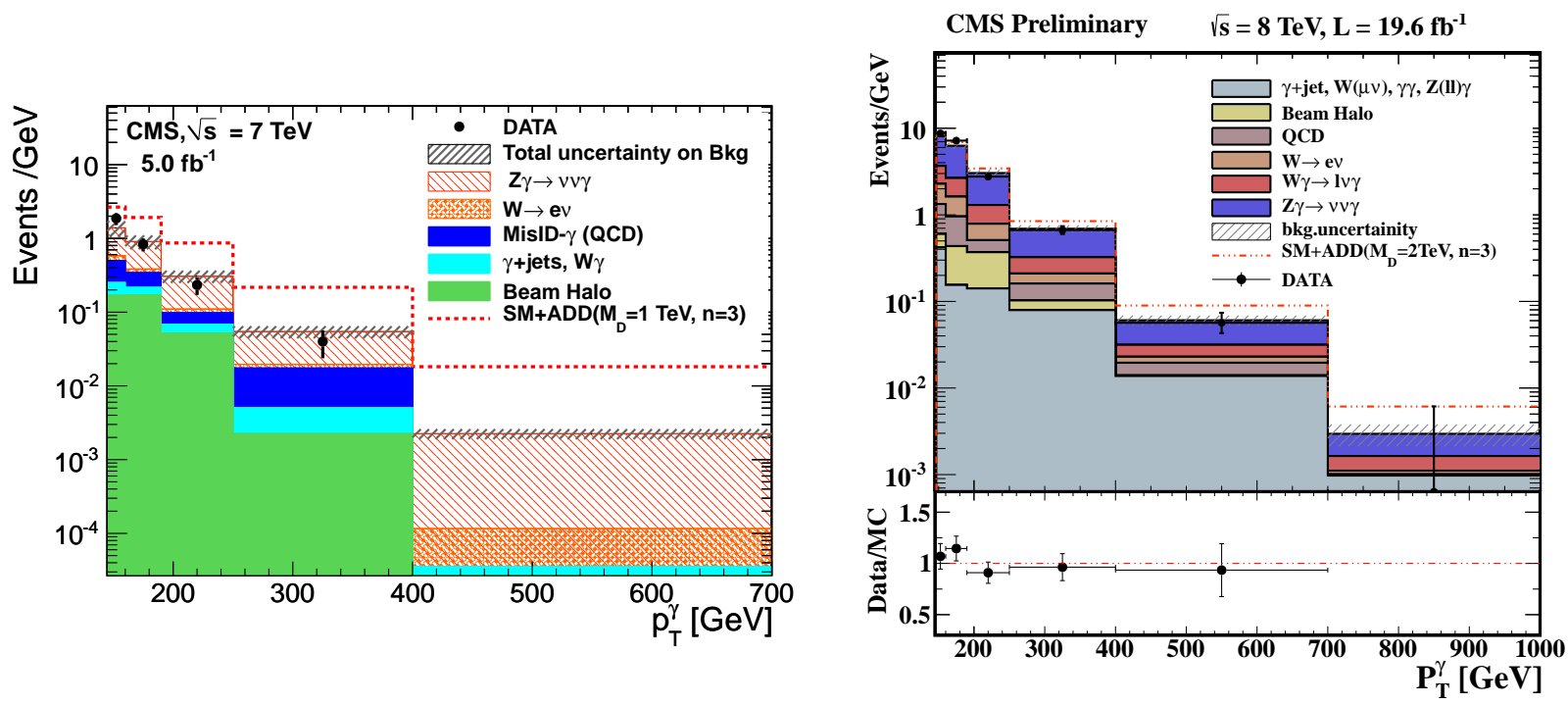

FIG. 2: $p_{T}(\gamma)$ distribution after full selection in the CMS mono-photon analysis:(left) at $\sqrt{s}=$ $7 \mathrm{TeV}$ using $5.0 \mathrm{fb}^{-1}$ of data [65], (right) at $\sqrt{s}=8 \mathrm{TeV}$ using $19.6 \mathrm{fb}^{-1}$ of data [66]. It should be noted that no WIMP signal contribution is plotted but the red dashed signal corresponds to a particular model for large extra dimensions. 65]

The ATLAS and the CMS collaborations have derived 90\% CL upper limit on WIMPnucleon cross section in the mono-photon final search. Figure 3 summarizes these results 
and comparisons with direct detection limits. For the spin-independent case, CMS considers the D5 operator while ATLAS uses D5 and the D1 operators. In the spin-dependent case both collaborations use D8 while ATLAS additionally studies the D9 operator. For spinindependent scenarios both collaborations obtain similar upper limits at $\sqrt{s}=7 \mathrm{TeV}$ while CMS results at $\sqrt{s}=8 \mathrm{TeV}$ provides the leading bound on WIMP-nucleon cross section across the entire DM mass range of $M_{\chi}=1-1000 \mathrm{GeV}$. In comparison to direct searches from LUX [58] and SuperCDMS [60] these limits improve only existing constraints for $M_{\chi}<3$ $\mathrm{GeV}$, while for $M_{\chi}>3 \mathrm{GeV}$ direct detection limits provide stronger bounds. In case of a spin-dependent coupling, the D9 results from ATLAS and D8 results from CMS provide similar and leading bounds up to $M_{\chi}$ of $200 \mathrm{GeV}$ of, while for even larger $M_{\chi}$ IceCube reaches best sensitivity. Bounds on the D8 operators also achieve stronger constraints to up to to about $M_{\chi} \sim 100 \mathrm{GeV}$ compared to direct detection experiments. It should be noted that direct detection limits from other experiments like, PICASSO, SIMPLE, XENON-100 provide most sensitive bounds on WIMP-nucleon cross section for various mass intervals in $M_{\chi}$ starting from a few $\mathrm{GeV}$ to $100 \mathrm{GeV}$, but generally these bounds are weakend in the spin-dependent case.

\section{B. Mono-jet searches}

ATLAS and CMS performed searches in the final state with a jet and missing transverse energy. The main backgrounds to this analysis are $Z \rightarrow \nu \nu, W+$ jets, single top, top pair, diboson and multijet events, whereas $Z \rightarrow \nu \nu$ is an irreducible background.

The ATLAS mono-jet analysis was performed using a luminosity of $4.7 \mathrm{fb}^{-1}$ of data recorded at $\sqrt{s}=7 \mathrm{TeV}$ in 2011 and updated with $10.5 \mathrm{fb}^{-1}$ at a center of mass energy of $\sqrt{s}=8 \mathrm{TeV}$ recorded in 2012 [67, 68]. Both analyses utilize similar event selections. Events

are required to pass an $\mathbb{E}_{\mathrm{T}}$ based trigger of at least $\mathrm{E}_{\mathrm{T}}>80 \mathrm{GeV}$, with an efficiency of about $95 \%$ for $\mathbb{E}_{\mathrm{T}}>120 \mathrm{GeV}$. All events are required to pass the latter $\mathrm{E}_{\mathrm{T}}$ criterion. Events are also required to contain one jet with $p_{T}$ (jet) $>120 \mathrm{GeV}$ and $|\eta|<2.0$. Events with more than two additional jet with $p_{T}$ (jet) $>30 \mathrm{GeV}$ and $|\eta|<4.5$ are rejected. Multijet events which may pass the event selection due to the mis-measurement of one of the jets gives rise to (fake) $\mathbb{E}_{\mathrm{T}}$. Those are suppressed by ensuring that the sub-leading jet is well separated from the direction of $\mathrm{E}_{\mathrm{T}} . W / Z$ production is suppressed by applying a veto on 


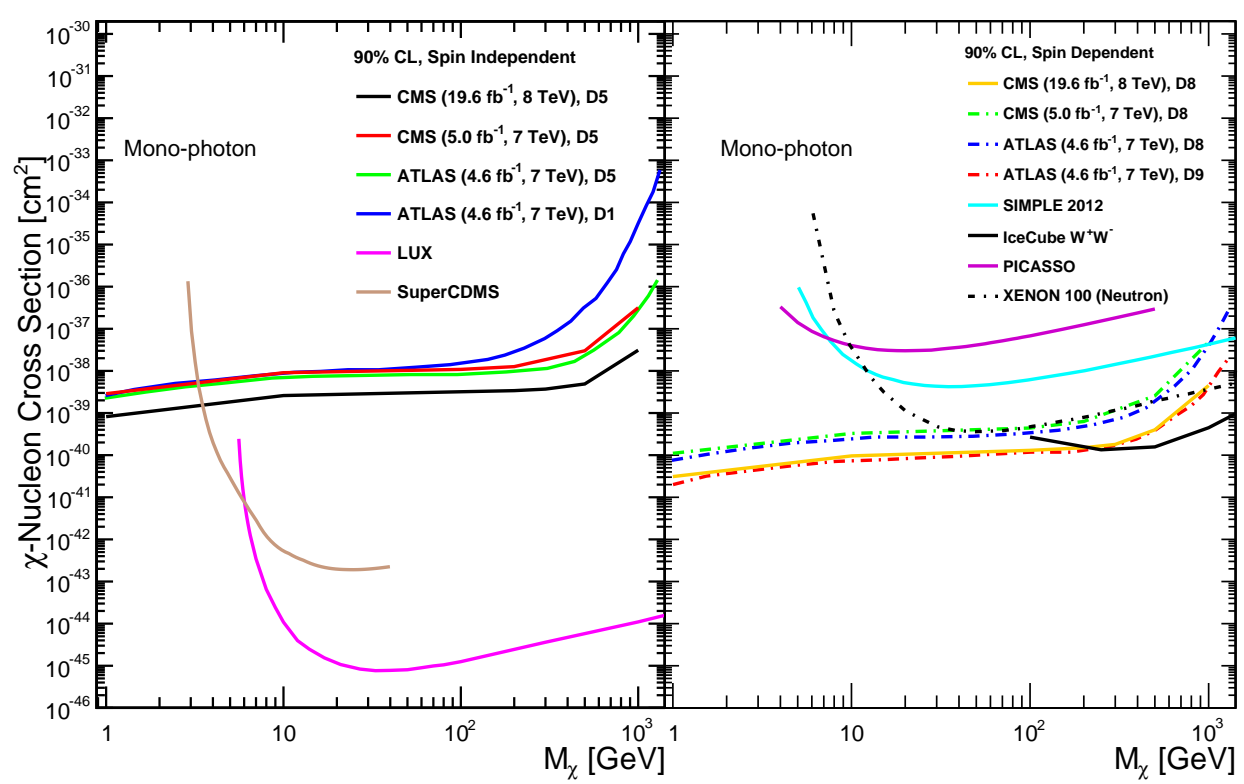

FIG. 3: Observed 90\% CL upper limit for spin-independent(left) and spin-dependent(right) DMnucleon cross section for mono-photon final state from the ATLAS and the CMS collaboration. The results are also compared with latest direct detection bound from LUX [58], SuperCDMS 60], XENON-100 63], IceCube 661, PICASSO [62], and SIMPLE [59].

electrons and muons.

Background from $Z \rightarrow \nu \nu$ is estimated using a $Z \rightarrow \ell \ell$ control region and a transfer functions to account for kinematic differences of background contributions in the hadronic final state. A similar procedure is employed for $W+$ jets production. Top and diboson backgrounds are taken from Monte Carlo simulations. Multijet production is estimated from data by an enriched selection of this process. Figure 4 shows $\mathbb{E}_{\mathrm{T}}$ and leading jet $p_{T}$ of the signal region requiring $\mathrm{E}_{\mathrm{T}}, p_{T}(\mathrm{jet})>120 \mathrm{GeV}$. The search is performed in 4 signal regions, defined by lower bounds on the leading jet momentum and $\mathbb{E}_{\mathrm{T}}$ with values of $120,220,350$ and $500 \mathrm{GeV}$, respectively. None of the signal regions show any significant excess over expected background.

The CMS mono-jet analysis was performed using $4.7 \mathrm{fb}^{-1}$ of $\sqrt{s}=7 \mathrm{TeV}$ data and $19.5 \mathrm{fb}^{-1}$ of $\sqrt{s}=8 \mathrm{TeV}$ [51, 69]. The data used to study events with a single jet and missing transverse energy are collected using a combination of jet and $\mathbb{E}_{\mathrm{T}}$ triggers. The $\mathrm{E}_{\mathrm{T}}$ trigger uses a threshold of $80(120) \mathrm{GeV}$ for the 7 (8) TeV analysis. All triggers are fully 

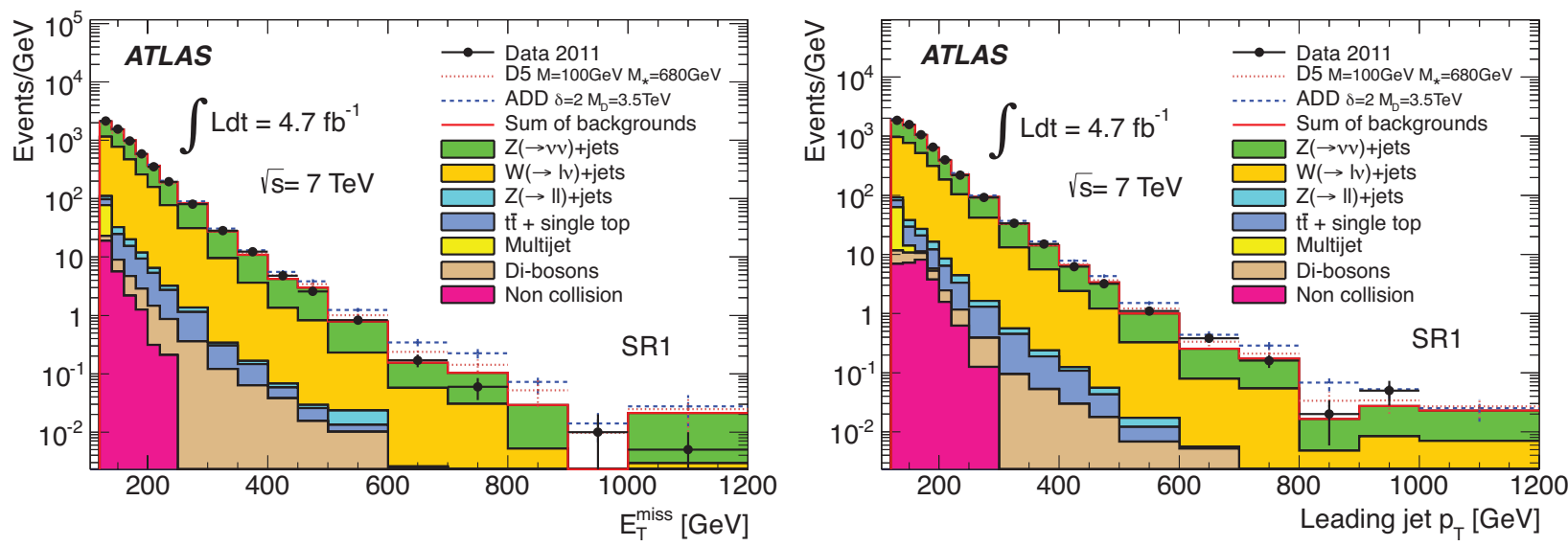

FIG. 4: $\mathrm{E}_{\mathrm{T}}$ (left) and transverse momentum of the leading jet (right) in loosest signal region of the ATLAS monojet search [67, 68].

efficient in the selected signal regions. Events are selected by requiring $\mathrm{E}_{\mathrm{T}}>200 \mathrm{GeV}$ and that the momentum of the leading jet exceeds $p_{T}($ jet $)>110 \mathrm{GeV}$. Events containing a second jet with $p_{T}$ (jet) $>30 \mathrm{GeV}$ are accepted, but any event with more jets of $p_{T}($ jet $)>30 \mathrm{GeV}$ are rejected. To suppress multijet background the angular separation between leading and sub-leading jets has to be less than $\Delta \phi\left(j_{1}, j_{2}\right)<2.5$ and also leptons with reconstructed leptons. In contrast to the ATLAS analysis even with isolated tracks $p_{T}(t r k)>10 \mathrm{GeV}$ are also rejected. All backgrounds except $Z \rightarrow \nu \nu+$ jets and $W+$ jets are modeled using MC simulation. These data driven backgrounds are modeled using signal regions dominated by $W \rightarrow \mu+$ jet and cross checked using the statistically limited $Z \rightarrow \mu \mu$ process for $Z \rightarrow \nu \nu$.

Various signal regions in $\mathrm{E}_{\mathrm{T}}$ between $\mathrm{E}_{\mathrm{T}}>250-550 \mathrm{GeV}$ are studied. Final $\mathrm{E}_{\mathrm{T}}$ selection for DM production is $\mathrm{E}_{\mathrm{T}}>350(400) \mathrm{GeV}$ in the $7 \mathrm{TeV}(8 \mathrm{TeV})$ analysis. The $\mathrm{E}_{\mathrm{T}}$ distributions for both analyses are shown in Figure 5.

Mono-jet searches at LHC have been performed at $\sqrt{s}=7$ and $8 \mathrm{TeV}$ and might be expected to provide the strongest bounds on WIMP-nucleon cross section due to the fact that the rate of gluon or quark initial-state radiation is large relative to photon, $W$ or $Z$ boson radiation. The systematic uncertainties associated with this final state, however, are more severe than for more clean channels. Both the CMS and the ATLAS collaboration have considered D5 and D11 operators for spin-independent, and the D8 operator for spindependent coupling. In addition ATLAS has evaluated limits for D9 which are slightly stronger bounds on the scattering cross section. For the spin-independent couplings CMS 

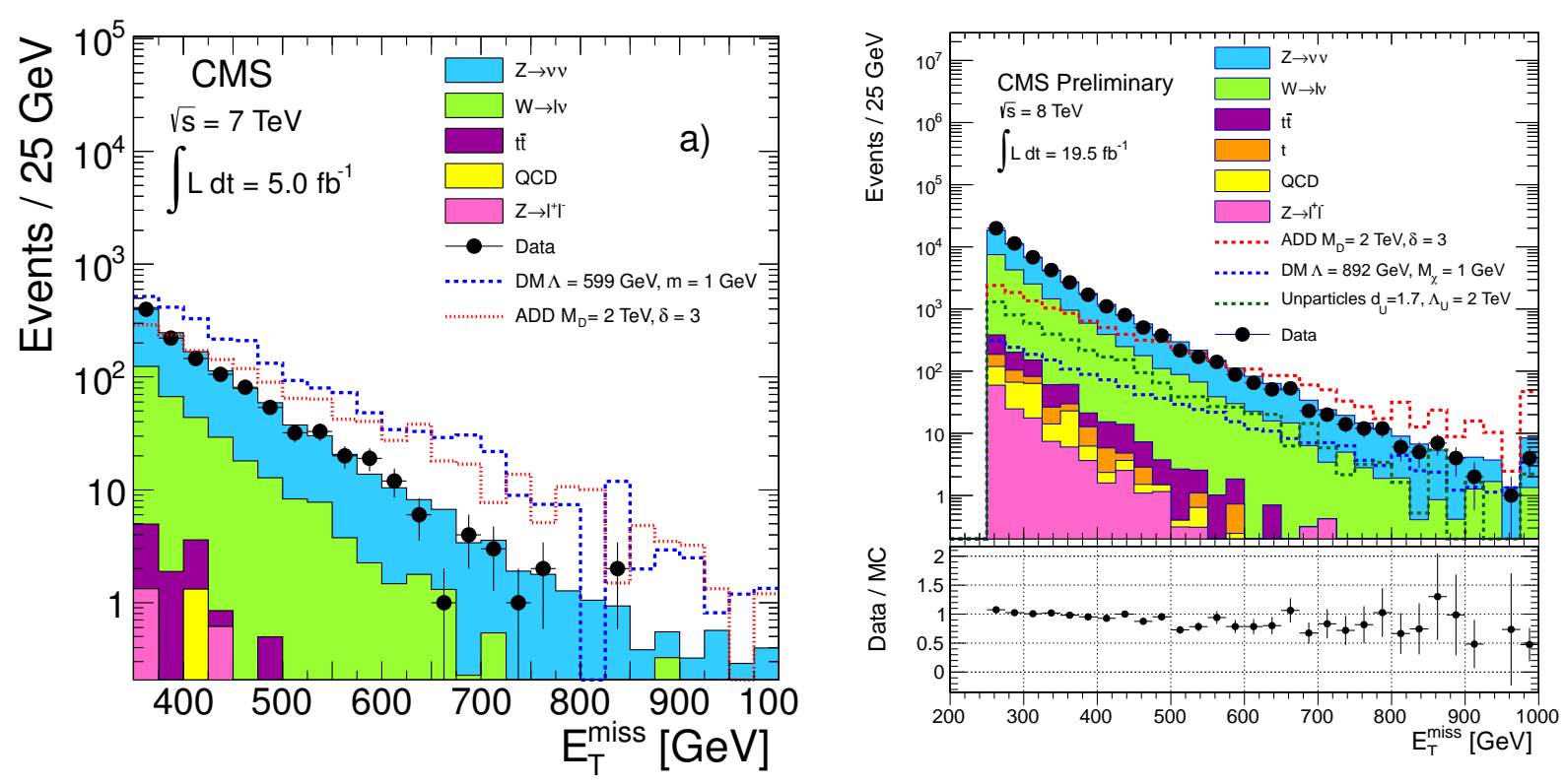

FIG. 5: $\mathrm{E}_{\mathrm{T}}$ distribution in the signal region of the CMS monojet analysis for $\sqrt{s}=7$ (left) and $\sqrt{s}=8$ (right). A simulated dark matter signal for axial-vector couplings and $m_{\chi}=1 \mathrm{GeV}$ is shown as dashed blue line. [51, 69]

results for D11 present the best upper limits for $M_{\chi}<10 \mathrm{GeV}$ while for rest of $M_{\chi}$ masses LUX has stronger upper bounds. CMS also has the leading collider bounds on mono-jets from the D5 operator, stronger than direct detection searches for $M_{\chi}<3 \mathrm{GeV}$.

As discussed in Sec. II it is generally assumed that limits obtained with collider-accessible mediator mass are weaker than the effective field theory. CMS has also shown results with limits obtained for different mediator mass $(M)$ and widths $(\Gamma)$. These results are shown in Figure 7. As the mass of the mediator approaches the kinematic range, the production cross section shows resonant enhancement and indicates on-shell production. As expected, when the mediator is light compared to the kinematic scale of the events, the resulting bounds grow weak quickly. The limits obtained for the vector couplings from these variations in mass and widths show that for heavy mediator mass they are approximately same as obtained from effective theory (EFT) framework [19, 49]. For mediator mass range from few hundreds of $\mathrm{GeV}$ to few $\mathrm{TeV}$, EFT limits are weaker due to an enhancement in the production cross section. In the region below a few hundreds $\mathrm{GeV}$ these limits are too strong. Figure 7 also shows that limits for heavy mediator are stronger relatively at lower values of $M_{\chi}$ which is complementary to the direct detection limits.

The lower limits on $\Lambda$ from collider searches can also be expressed in terms of relic 


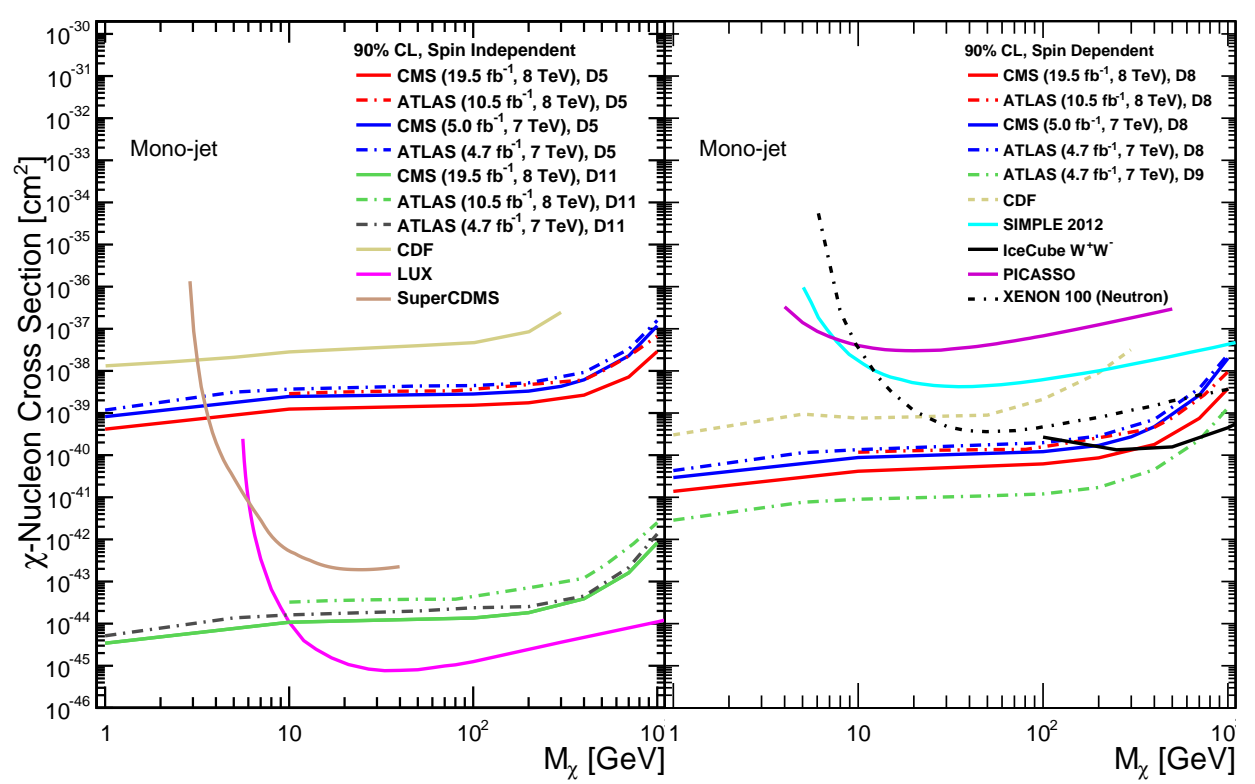

FIG. 6: Observed 90\% CL upper limit for spin-independent(left) and spin-dependent(right) DMnucleon cross section for mono-jet final state from the ATLAS, CMS, and CDF [22] collaboration. The results are also compared with latest direct detection bounds from LUX [58], SuperCDMS [60], XENON-100 63], IceCube 61], PICASSO 62], and SIMPLE [59].

abundance of WIMP production as described in Ref. [19, 70]. The ATLAS collaboration has translated their results of the mono-jet search at $\sqrt{s}=7 \mathrm{TeV}$ to WIMP annihilation rate into four light flavors of quark assuming equal coupling strength for all of them. These annihilation rates are estimated by translating vector and axial-vector limits as described in [19]. The annihilation rate is defined as $\langle\sigma v\rangle$ where $\sigma$ is the cross section and $v$ is the average relative velocity of dark matter. The limits are based on the assumption of $100 \%$ branching ratio of WIMPs to four light flavour of quarks. These results are summarized in Fig. 8. The figure also shows a comparison with the observations of Galactic satellite galaxies with the Fermi-LAT experiment [71] for Majorana WIMPs. For WIMPs to make up the relic abundance, annihilation rate must be above the thermal relic value observed by WMAP( dashed line) [72]. 


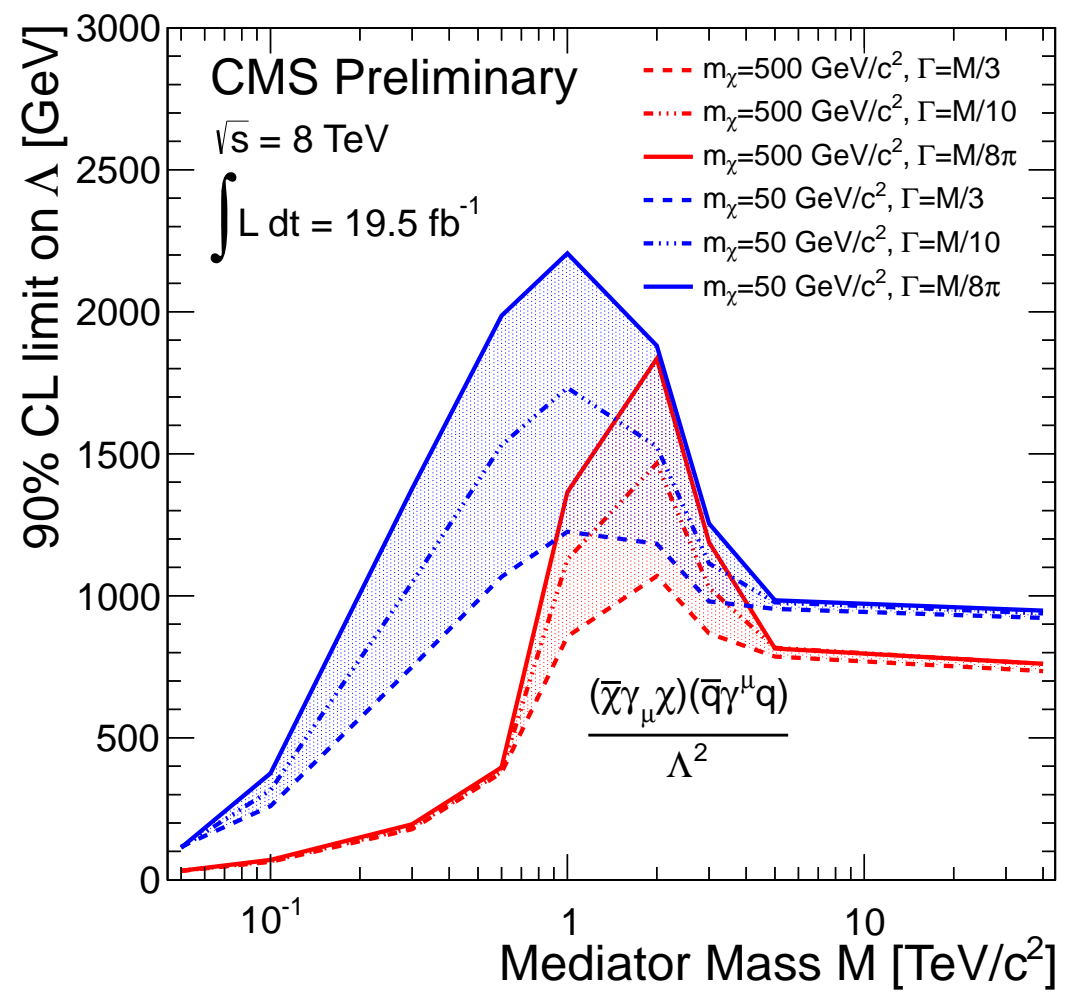

FIG. 7: 90\% CL observed limit on $\Lambda$ as a function of mass of the mediator(M) for two different $M_{\chi}$ values of $50 \mathrm{GeV}$ and $500 \mathrm{GeV}$. The widths are also varied from $\mathrm{M} / 3$ and $\mathrm{M} / 8 \pi$.

\section{Mono- $W / Z$ searches}

The ATLAS collaboration has performed searches for DM production with $W$ or $Z$ boson in their final state, probing either the hadronic or the leptonic decay mode of the $Z$. The CMS experiment also performed a search for DM production with the $W$ boson decaying leptonically.

1. $W / Z \rightarrow j e t+\mathbb{E}_{\mathrm{T}}$

The ATLAS collaboration is searching for dark matter pair production in association with a $W$ or $Z$ decaying hadronically using $20.3 \mathrm{fb}^{-1}$ of data at $\sqrt{s}=8 \mathrm{TeV}$ [73]. In contrast to other searches presented that assume equal couplings of the dark matter particles to uptype and down-type quarks, as they are not sensitive to a relative sign between those two couplings. For $W$ boson radiation there is interference between the diagrams in which the $W$ boson is radiated from the $u$ quark or the $d$ quark. In the case of equal couplings, the 


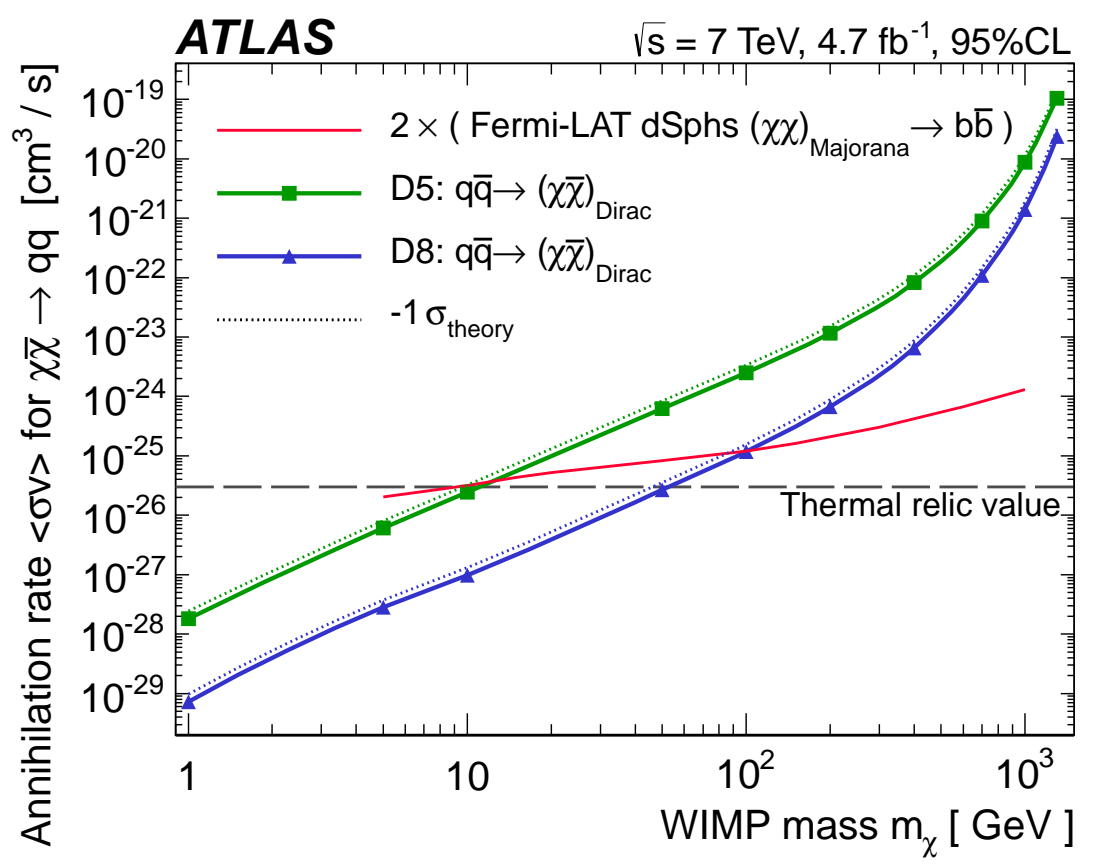

FIG. 8: 90\% CL observed limit on WIMP annihilation rates $\langle\sigma v\rangle$ as a function of $M_{\chi}$ (solid line). The impact of theoretical uncertainties on observed limits is shown as dotted lines.

interference is destructive and gives a small $W$ boson emission rate. However, if the up-type and down-type couplings are of opposite sign, one finds constructive interference and $W$ boson emission becomes the dominant process, even over radiation of photons or gluons.

This search uses particularly wide jets using the Cambridge-Aachen-Algorithm [74] with wide jet radii of $R=1.2$. These jets are intended to capture the full decay products of hadronically decaying $W$ and $Z$ bosons. The internal structure of these wide-radius jets is probed in terms of the momentum balance of their leading two constituents and the jet mass is calculated. Are are reconstructed using the anti $-k_{T}$ algorithm using $R=0.4$.

Events are recorded using a $\mathrm{E}_{\mathrm{T}}$ trigger that is fully efficient at the minimum requirement of $\mathrm{E}_{\mathrm{T}}>150 \mathrm{GeV}$. Events have to contain at least one large-radius jet of $p_{T}($ jet $)>250 \mathrm{GeV}$ and $\mid \eta($ jet $) \mid<1.2$. The jet mass should be compatible with a $W$ or $Z$ boson, $50<m($ jet $)<$ 
$120 \mathrm{GeV} \cdot \sqrt{y}>0.4$ is required to suppress backgrounds from non-hadronic $W$ or $Z$ boson decays. Two signal regions are defined using $\mathbb{E}_{\mathrm{T}}>350$ and $500 \mathrm{GeV}$. Events with more than one narrow jet of $p_{T}>40 \mathrm{GeV}$ are rejected if the narrow jets do not overlap with the wide radius jet or if any narrow jet has $\Delta \phi\left(\mathbb{E}_{\mathrm{T}}, j\right)<0.4$. Any event containing a reconstructed photon or lepton (electron or muon) candidate with $p_{T}>10 \mathrm{GeV}$ in the fiducial detector region are rejected as well.

Dominant backgrounds to this selection are $Z \rightarrow \nu \nu+$ jet production and $W / Z+$ jets production with leptonic decays in which the lepton was not reconstructed. These backgrounds are estimated using data control regions with an inverted muon veto. Diboson, singleand double-top production are taken from simulations. Multijet production is negligible as background.

After full selection no significant excess is observed for either signal region. Figure 9 shows the $m$ (jet) distribution for both signal regions.

2. $W \rightarrow \ell \nu+\mathbb{E}_{\mathrm{T}}$

A search for dark matter production in final states with an electron or a muon and a neutrino has been performed by the CMS collaboration using $20 \mathrm{fb}^{-1}$ of data recorded at $\sqrt{s}=8 \mathrm{TeV}[75]$. The results can be interpreted in terms of the cross section of a $W$-boson recoiling against a pair of dark matter particles in an effective theory, considering vectorand axial-vector like couplings. Also varying coupling strength to up and down type quarks is assumed, parametrized by $\xi$ with $\xi=0, \pm 1$.

Candidate events are recorded using single-lepton trigger. The reconstructed momentum required for muons is $p_{T}(\mu)>45 \mathrm{GeV}$ and for electrons is $p_{T}(e)>100 \mathrm{GeV}$. Further criteria are applied by requiring $0.4<p_{T}(\ell) / \mathbb{E}_{\mathrm{T}}<1.5$ and an angular separation between lepton and $\mathbb{E}_{\mathrm{T}}$ of $\Delta \phi(\ell, \nu)>0.8 \pi$. The main observable in this analysis is the transverse mass of the lepton- $\mathbb{E}_{\mathrm{T}}$ system:

$$
M_{T}=\sqrt{2 \cdot p_{T}(\ell) \cdot \mathrm{E}_{\mathrm{T}} \cdot(1-\cos \Delta \phi(\ell, \nu)}
$$

The dominant background is the high transverse mass tail of the $W \rightarrow \ell \nu$ decay. Further background arise from multijet, top-quark, $Z$ boson and diboson production. All backgrounds are modeled using simulations. Due to low statistics at very high $M_{T}$ the full $M_{T}$ 


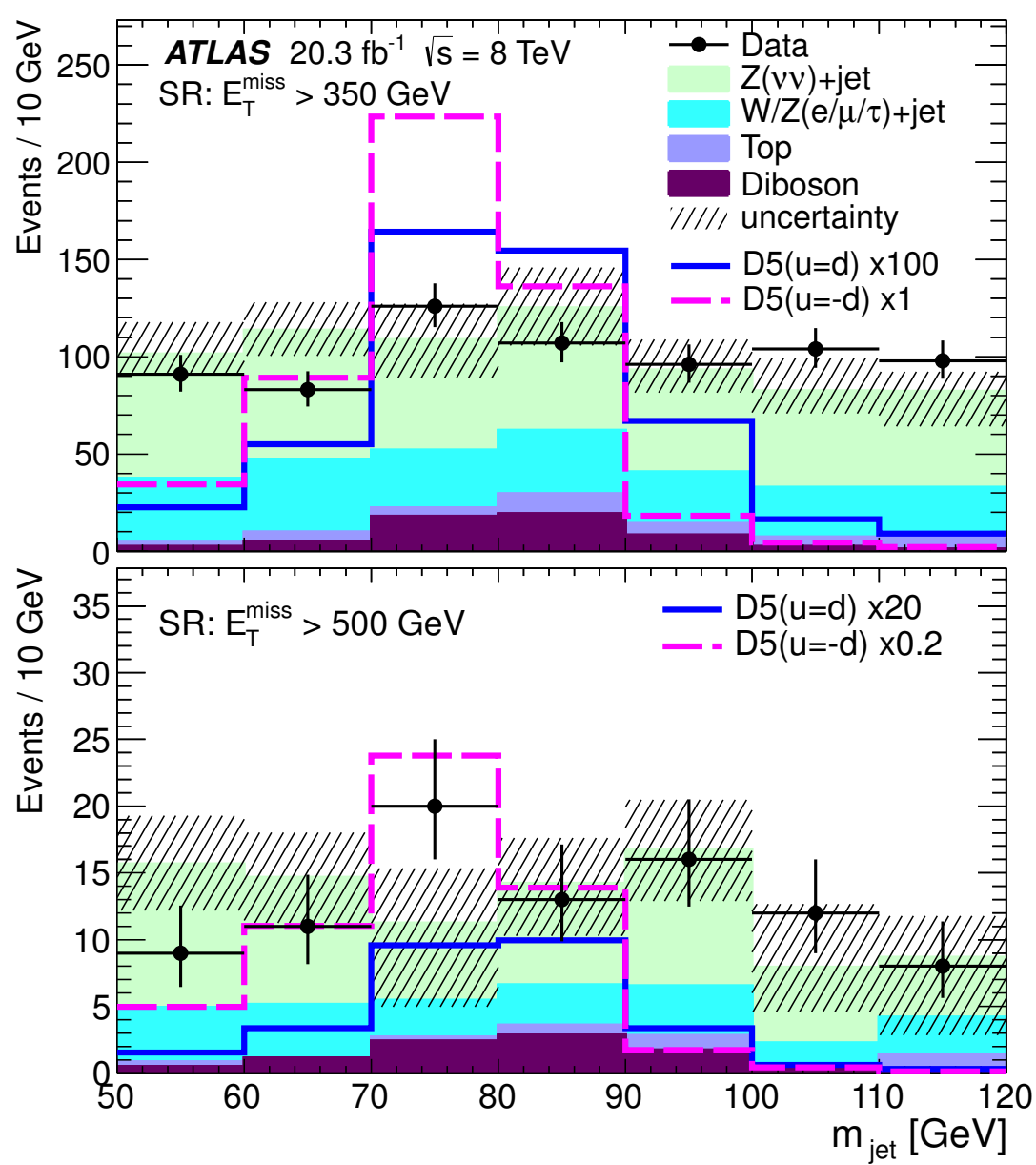

FIG. 9: Distribution of $m$ (jet) in data and for the predicted background in the signal regions (SR) with $\mathrm{E}_{\mathrm{T}}>350 \mathrm{GeV}$ (top) and $\mathrm{E}_{\mathrm{T}}>500 \mathrm{GeV}$ (bottom). Also shown are the combined mono- $W$-boson and mono- $Z$-boson signal distributions with $m_{\chi}=1 \mathrm{GeV}$ and $M_{*}=1 \mathrm{TeV}$ for the $D 5$ destructive and $D 5$ constructive cases, scaled by factors defined in the legends. Uncertainties include statistical and systematic contributions. [73]

distribution is fit using an empirical function and extrapolated to the region of interest. After full selection again no significant deviation between background expectation and data is observed. Figure 10 shows the $M_{T}$ distributions for the electron and muon channels along with different couplings for up/down type quarks for DM production.

\section{3. $Z \rightarrow \ell \ell+\mathrm{E}_{\mathrm{T}}$}

The search for dark matter production in association with a $Z$ boson decaying to two leptons has been performed by the ATLAS collaboration using $20.3 \mathrm{fb}^{-1}$ of data collected at 

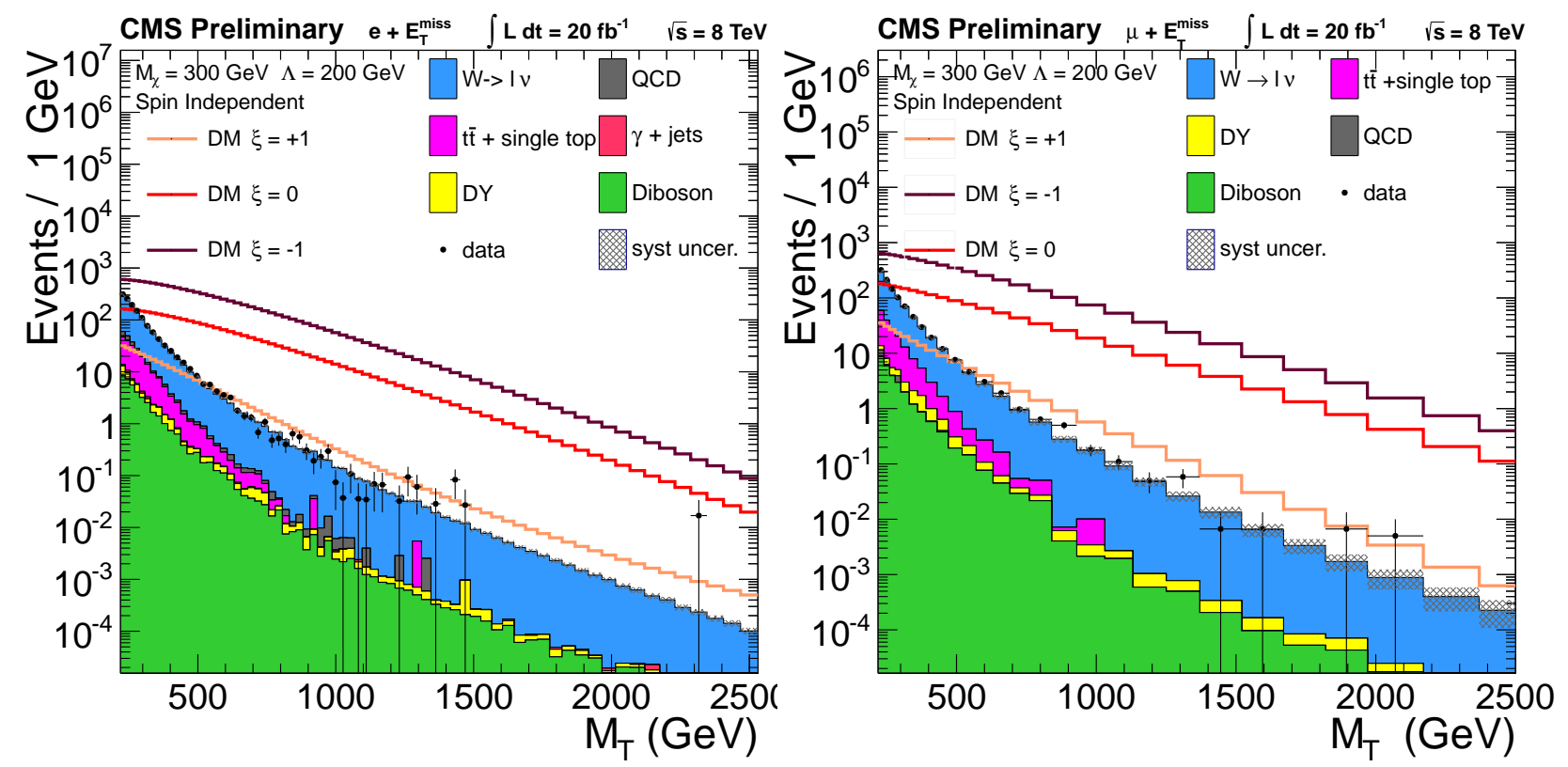

FIG. 10: Transverse mass distribution for the electron (left) and muon (right) channel. The shaded area represents the total systematic uncertainty on the SM background. Simulated signal distributions for a $\mathrm{M}=300 \mathrm{GeV},=200 \mathrm{GeV}$ and $=1,0$ are also shown. [75]

$\sqrt{s}=8 \mathrm{TeV}[76$. The data were recorded using a combination of single and di-lepton triggers. Events were selected if they contained two electrons or muons with $p_{T}(\ell)>20 \mathrm{GeV}$ and invariant mass $76<m(\ell \ell)<106 \mathrm{GeV}$ forming a $Z$ boson candidate. To suppress events with $\mathbb{E}_{\mathrm{T}}$ from mis-measured jets $\Delta \phi\left(\mathbb{E}_{\mathrm{T}}, p_{T}(\ell \ell)\right)>2.5$ is required. The absolute value of the pseudorapidity of the dilepton system is required to be $\eta(\ell \ell)<2.5$, and $\left|p_{T}(\ell \ell)-\mathrm{E}_{\mathrm{T}}\right| / p_{T}(\ell \ell)<0.5$, where $p_{T}(\ell \ell)$ is the transverse momentum of the dilepton system. Events are removed if they contain a jet with $p_{T}((j e t))>25 \mathrm{GeV}$ or a third lepton with $p_{T}(\ell)>7 \mathrm{GeV}$. Four inclusive signal regions with $\mathrm{E}_{\mathrm{T}}>150,250,350$, and $400 \mathrm{GeV}$ are defined.

The dominant background is diboson production. $W Z$ and $Z Z$ are estimated using simulation; $W W, t \bar{t}, W t, Z \rightarrow \tau \tau$ and $W / Z+$ jets are estimated from data. No significant excess over background expectation is observed after full selection as seen in Fig. 11.

The production of a DM pair in association with a $W$ or $Z$ boson is different with respect to the photon or jet cases as the sign of the DM coupling to up-type and down-type quarks play an important role in production rate, hence limits are also sensitive to these couplings. Figure 12 summarizes these searches from the CMS and the ATLAS collaborations for 


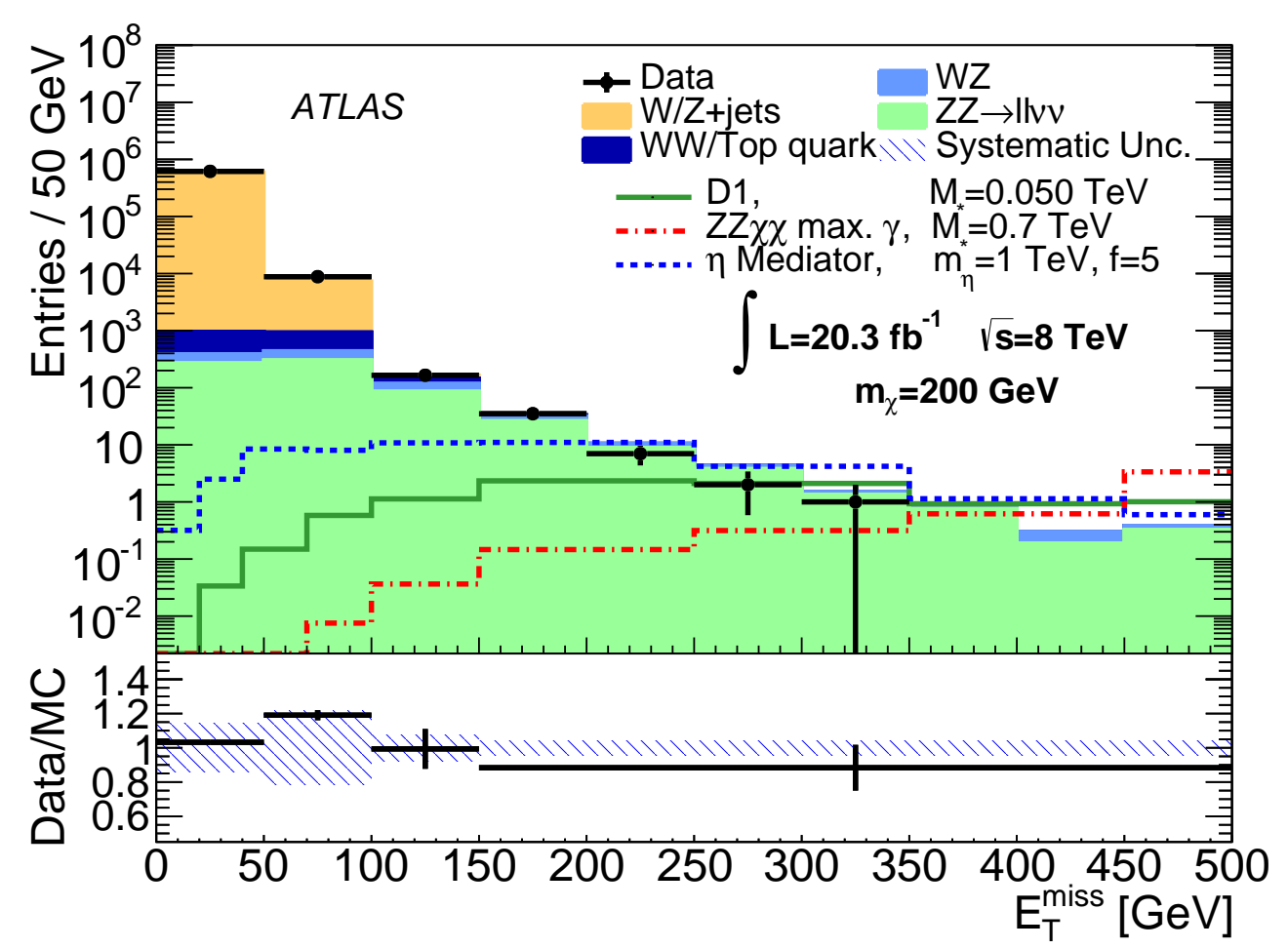

FIG. 11: $E_{\mathrm{T}}$ distribution of the ATLAS mono- $Z$ with $Z \rightarrow \ell \ell$ search [76].

different mono-W/Z final states.

For $W \rightarrow l \nu$ decay the strongest observed bound for spin-independent and spin-dependent couplings are from the ATLAS collaboration with D5( $\xi=-1)$ and D9 operators. It should be noted that the ATLAS limits are evaluated for 95\% CL. The D1 case is much more weakly bounded, as are cases where the couplings to up- and down-type quarks do not lead to such strong constructive interference, while bounds on D8 comparable to those from spin-dependent direct detection.

Similarly for $W / Z \rightarrow$ jj decay mode $\mathrm{D} 5(\xi=-1)$ and $\mathrm{D} 9$ gives the best limits. The $Z \rightarrow \ell \ell$ final state is analyzed by the ATLAS collaboration with D1 and D9 giving the strongest observed 95\% CL upper limits for spin-independent and spin-dependent couplings respectively. The most stringent bound among all the final states for mono- $\mathrm{W} / \mathrm{Z}$ production are from hadronic final states. Direct search limits from LUX are the strongest current bounds for $M_{\chi}>6 \mathrm{GeV}$ for any spin-independent scenario. The limits on the D9 operators are stronger than the direct detection bounds for the entire $M_{\chi}$ mass range accessible to the LHC for spin-dependent interactions. 


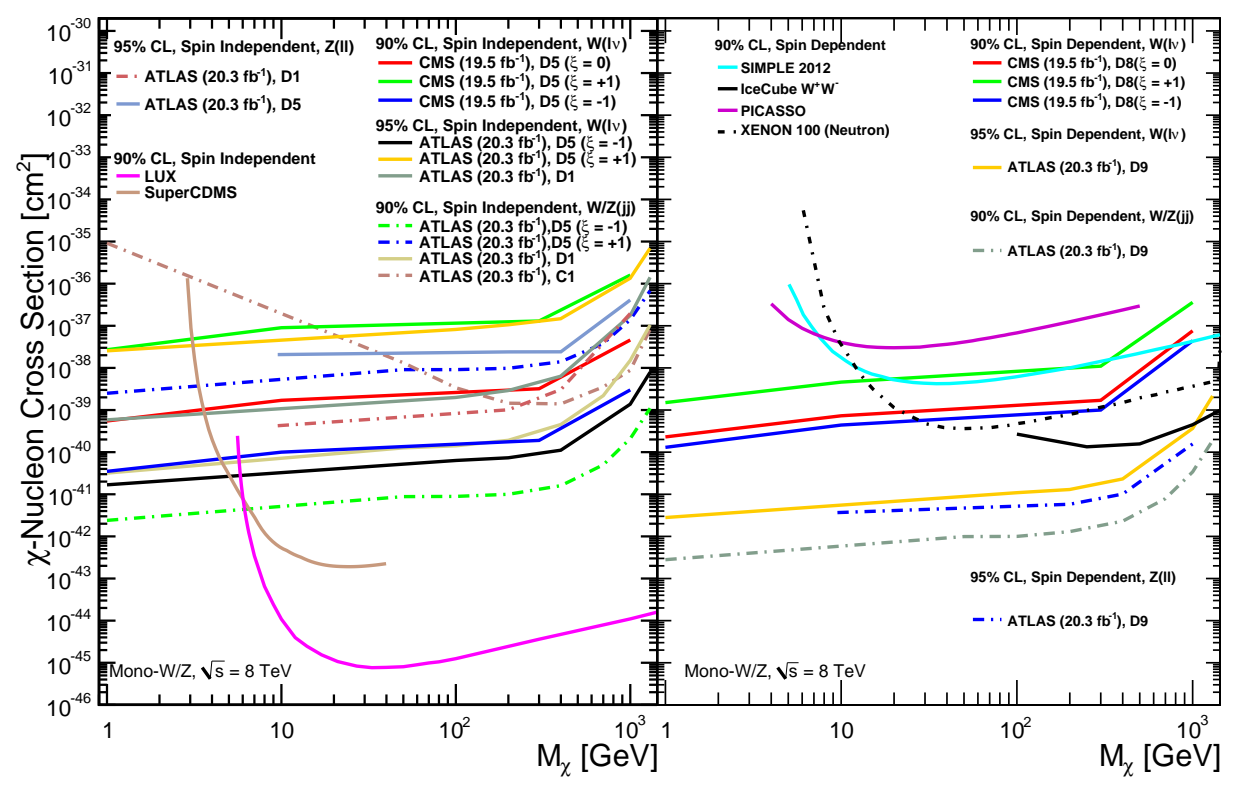

FIG. 12: Observed upper limits for spin-independent(left) and spin-dependent(right) DM-nucleon cross section for mono-W and mono-Z final state from the ATLAS and the CMS experiments. The results are also compared with latest direct detection bound from LUX [58], SuperCDMS [60], XENON-100 63], IceCube 61], PICASSO 62], and SIMPLE [59].

\section{Constraints from Higgs boson measurements}

Various models predict that a 'hidden' dark matter sector is coupled to the visible standard model via the Higgs sector, which allows for a renormalizable direct coupling. The Higgs boson then would decay into additional weakly interacting massive particles. This would subsequently be observable as a deviation from the expected Standard Model Higgs branching ratios. Measurements of Higgs boson properties and dedicated searches performed by the ATLAS and CMS collaborations lead to constraints on the $H \rightarrow$ invisible branching ratio and therefore couplings to dark matter particles.

\section{Limits for $Z H$ associated production}

Both the ATLAS and CMS collaborations have performed searches for the invisible decay of Higgs bosons using the associated production of a Higgs boson with a $Z$ boson. 
ATLAS uses the entire $2011 \sqrt{s}=7 \mathrm{TeV}\left(4.7 \mathrm{fb}^{-1}\right)$ and $2012 \sqrt{s}=8 \mathrm{TeV}\left(20.3 \mathrm{fb}^{-1}\right)$ datasets to search for anomalous decay of the Higgs boson in associated $Z H$ production [77]. Events with a dilepton pair (electron or muons) with an invariant mass of $76<m_{\ell \ell}<106 \mathrm{GeV}$ and $\mathbb{E}_{\mathrm{T}}>90 \mathrm{GeV}$ are selected. An additional robust version of the $\mathbb{E}_{\mathrm{T}}$ is defined for this analysis: $\left(\mathbb{E}_{\mathrm{T}}^{\mathrm{trk}}\right)$ which is the missing transverse momentum based on the reconstructed tracks. In order to suppress events in which $\mathbb{E}_{\mathrm{T}}$ arises from mismeasurements in the calorimeter $\mathbb{E}_{\mathrm{T}}$ and $\mathbb{E}_{\mathrm{T}}^{\text {trk }}$ are required to point in the same direction with $\Delta \phi\left(\mathbb{E}_{\mathrm{T}}, \mathbb{E}_{\mathrm{T}}^{\mathrm{trk}}\right)<0.2$. The $Z$ and the invisible decaying Higgs boson are expected to form a back-to-back topology, therefore the azimuthal opening angle between $\mathbb{E}_{\mathrm{T}}$ and the dilepton system is required to be $\Delta \phi\left(p_{T}^{\ell \ell}, \mathrm{E}_{\mathrm{T}}\right)>2.6$ and the angle between the lepton pair is required to be less than $\Delta \phi(\ell, \ell)<1.7$ due to the boost of the dilepton system. The momentum of the reconstructed $Z$ boson should be balanced by the invisibly decaying Higgs boson so $\left|\mathbb{E}_{\mathrm{T}}-p_{T}^{\ell \ell}\right| / p_{T}^{\ell \ell}<0.2$ is required. A maximum likelihood fit to the $\mathbb{E}_{\mathrm{T}}$ distribution is performed and no significant excess is observed. Limits on the cross section times branching ratio for a Higgs boson decaying to invisible particles are set for a mass range of $110<m_{H}<400 \mathrm{GeV}$ and converted to the WIMP-nucleon scattering cross section assuming Higgs portal dark matter and $m_{\chi} \leq m_{H} / 2$. Figure 13 presents the limits on the spin-independent WIMP-nucleon scattering cross section assuming Higgs portal dark matter.

The CMS collaboration searches for invisible decays of the Higgs boson using both associated $Z H$ production and vector boson fusion (VBF) production. The $Z H$ searches utilize final states with a pair of charged leptons (electrons or muons) or a b-quark pair $(b \bar{b})$ [78]. Analyses are based on $4.9 \mathrm{fb}^{-1}$ of $\sqrt{s}=7 \mathrm{TeV}$ data and $19.7 \mathrm{fb}^{-1}$ of $\sqrt{s}=8 \mathrm{TeV}$ data.

a. Associated $Z H$ production The dilepton channel requires a pair of isolated leptons with an invariant mass within $\pm 15 \%$ of the mass of the $Z$ boson. Events with any $b$-tagged jet or more than one light flavor jet are rejected. In the ATLAS analysis similar requirements are applied with $\mathbb{E}_{\mathrm{T}}>120 \mathrm{GeV}, \Delta \phi\left(p_{T}^{\ell \ell}, \mathbb{E}_{\mathrm{T}}\right)>2.7$ and $\left|\mathbb{E}_{\mathrm{T}}-p_{T}^{\ell \ell}\right| / p_{T}^{\ell \ell}<0.25$ to further increase the selection purity. The $\mathrm{E}_{\mathrm{T}}$ distribution obtained using the $Z(\rightarrow \ell \ell) H(\rightarrow i n v$.) selection is shown in Fig. 14.

The $Z(\rightarrow b \bar{b}) H(\rightarrow i n v$. $)$ uses an event selection requiring large $\mathbb{E}_{\mathrm{T}}$ and a $b$-quark pair consistent with the $Z$ boson mass. Events are recorded using $\mathbb{E}_{\mathrm{T}}$ and $\mathbb{E}_{\mathrm{T}}+$ jets triggers. In these events $\mathbb{E}_{\mathrm{T}}$ and $\mathbb{E}_{\mathrm{T}}^{\text {trk }}$ are required to point into the same direction 


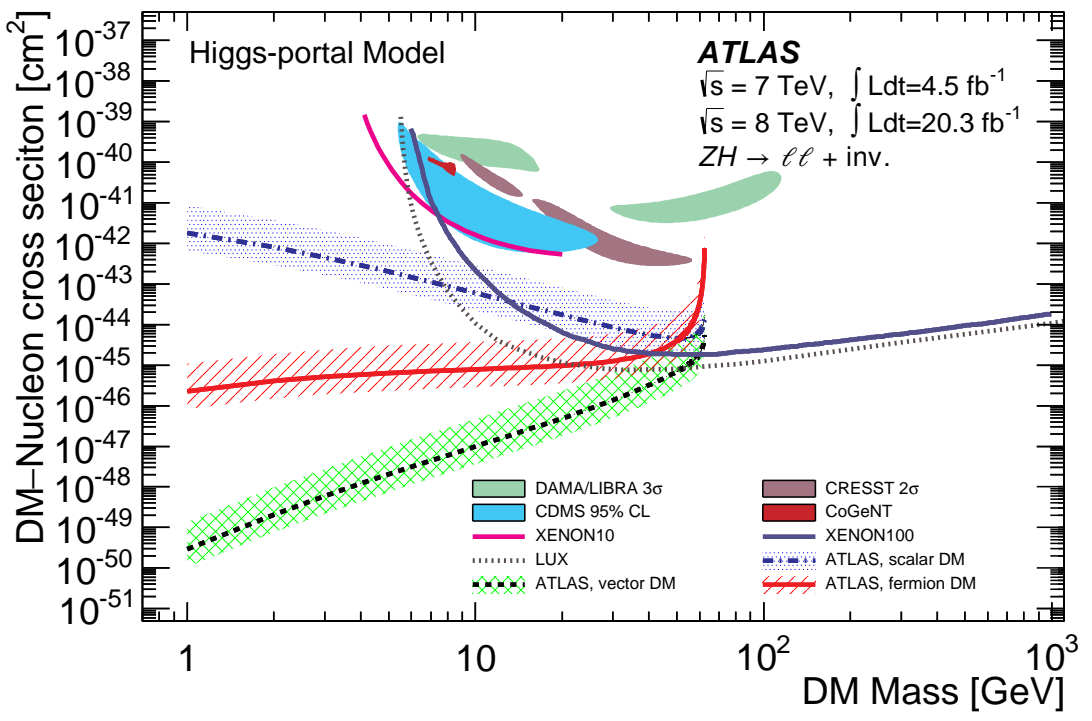

FIG. 13: Limits on WIMP-nucleon scattering cross section at 90\% CL, extracted from the $B R(H \rightarrow i n v$.$) limit in a Higgs-portal scenario, compared to direct DM searches [77]$

$\Delta \phi\left(\mathbb{E}_{\mathrm{T}}, \mathbb{E}_{\mathrm{T}}^{\mathrm{trk}}\right)<0.5$. The analysis is further divided into three regions, based on $\mathbb{E}_{\mathrm{T}}$, denoted "low" $\left(100<\mathrm{E}_{\mathrm{T}}<130 \mathrm{GeV}\right)$, "intermediate" $\left(130<\mathrm{E}_{\mathrm{T}}<170 \mathrm{GeV}\right)$, and "high" $\left(\mathbb{E}_{\mathrm{T}}>170 \mathrm{GeV}\right)$. The azimuthal separation between $\mathbb{E}_{\mathrm{T}}$ and the closest jet is required to be $\Delta \phi\left(\mathbb{E}_{\mathrm{T}}, j\right)>0.5$ for the "high" region and for "low" and "intermediate" regions $\Delta \phi\left(\mathrm{E}_{\mathrm{T}}, j\right)>0.7$ is required.

For the low mass region, the $\mathbb{E}_{\mathrm{T}}$ significance, the ratio of $\mathbb{E}_{\mathrm{T}}$ and the square root of the scalar sum of transverse energy of all particle-flow objects, is required to be greater than $3 \mathrm{GeV}^{\frac{1}{2}}$. Events with isolated leptons are rejected and the two jets forming the $Z$-candidate are required to be $b$-tagged. The analysis then uses a boosted decision tree (BDT) discriminator to separate signal candidates from background events. The BDT is trained using simulated samples for signal and all background processes after the full selection described above. This is performed separately for each Higgs boson mass considered. Figure 14 shows the $\mathbb{E}_{\mathrm{T}}$ and BDT output for $Z H$ analyses.

b. Vector Boson Fusion The VBF topology is distinguished by two jets in opposite forward directions. The events are recorded with a dedicated trigger for this topology, requiring $\mathrm{E}_{\mathrm{T}}>65 \mathrm{GeV}$, requiring the transverse momentum of the forward jets to be $p_{T}>40 \mathrm{GeV}$, and requiring that the invariant mass of the forward jets be $M(j j)>800 \mathrm{GeV}$. This selection is further tightened offline by requiring $p_{T}($ jets $)>50 \mathrm{GeV}, \mid \eta($ jet $) \mid<4.7$, 

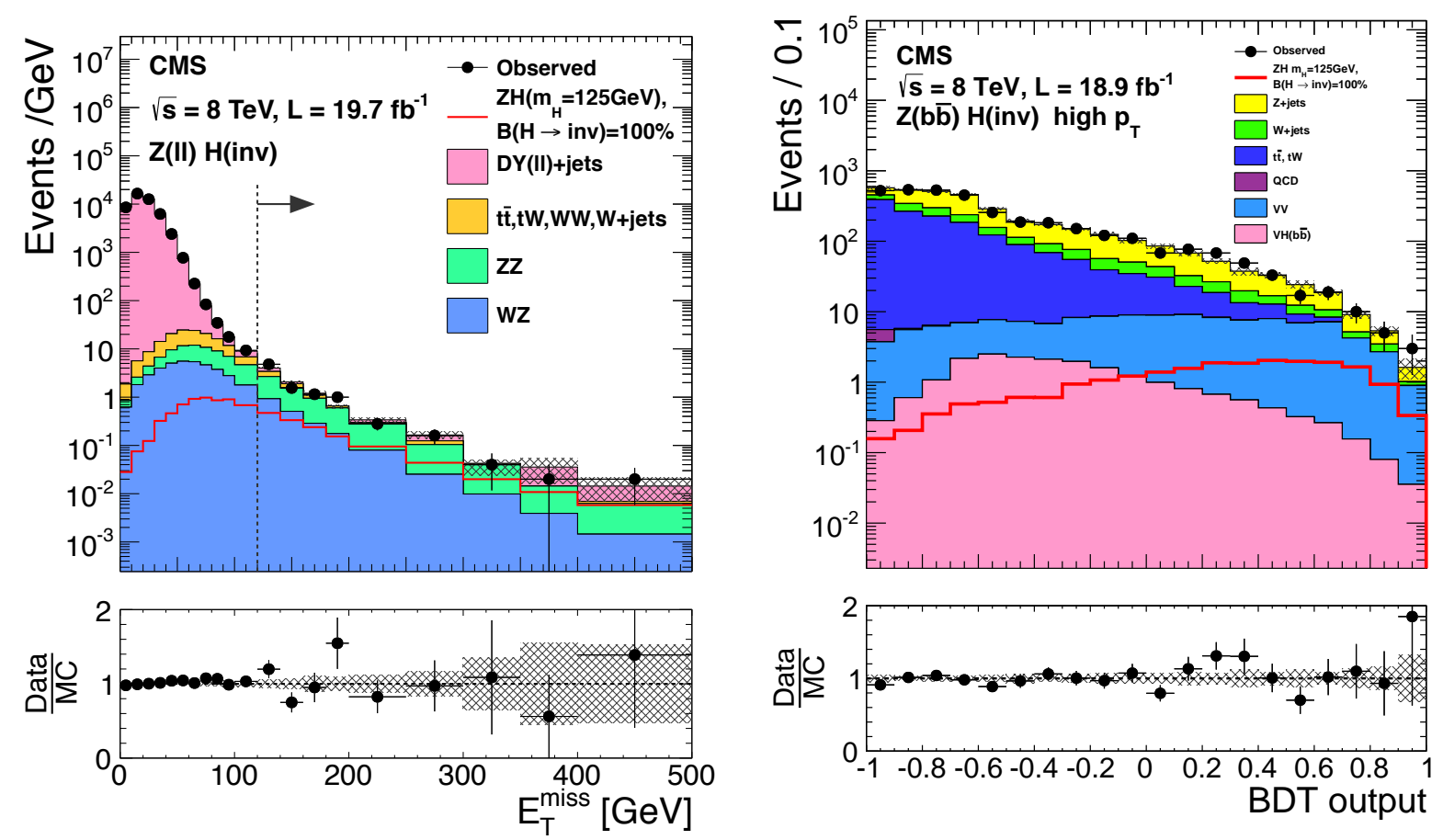

FIG. 14: Main discriminating variables in their final selection for the $Z(\rightarrow \ell \ell) H($ left $)$ and $Z(\rightarrow$ $b \bar{b}) H$ (right, high $p T$ region is shown) analyses. [78]

$\eta\left(j_{1}\right) \cdot \eta\left(j_{2}\right)<0, \Delta \eta\left(j_{1}, j_{2}\right)>4.2, \mathrm{E}_{\mathrm{T}}>130 \mathrm{GeV}$, and $M(j j)>1100 \mathrm{GeV}$. Multijet backgrounds are reduced by requiring $\Delta \phi(j j)<1.0$ and events are removed if there are any jets with $p_{T}>30 \mathrm{GeV}$ between the two leading forward jets. Figure 15 shows the $\mathrm{E}_{\mathrm{T}}$ distribution after the full selection is applied. Dominant backgrounds in this channel are $Z \rightarrow \nu \nu+$ jets and $W+$ jets whereas for the later the charged lepton is mis-reconstructed. These backgrounds are estimated from data control regions. Smaller background processes of single-top, top-pair and diboson production are estimated using simulations.

None of these searches yield any significant deviation from the background expectation and upper limits are set. These limits again are interpreted in the context of a Higgs-portal model as limit on the WIMP-nucleon scattering cross section for scalar, vector and fermionic dark matter candidates. Figure 16 shows the upper limits at 90\% CL on the WIMP-nucleon cross section as a function of the DM mass, derived from the upper limit on $\mathcal{B R}(H \rightarrow$ inv. $)$ for $m_{H}=125 \mathrm{GeV}$, in the scenarios where the DM candidate is a scalar, a vector, or a Majorana fermion. 


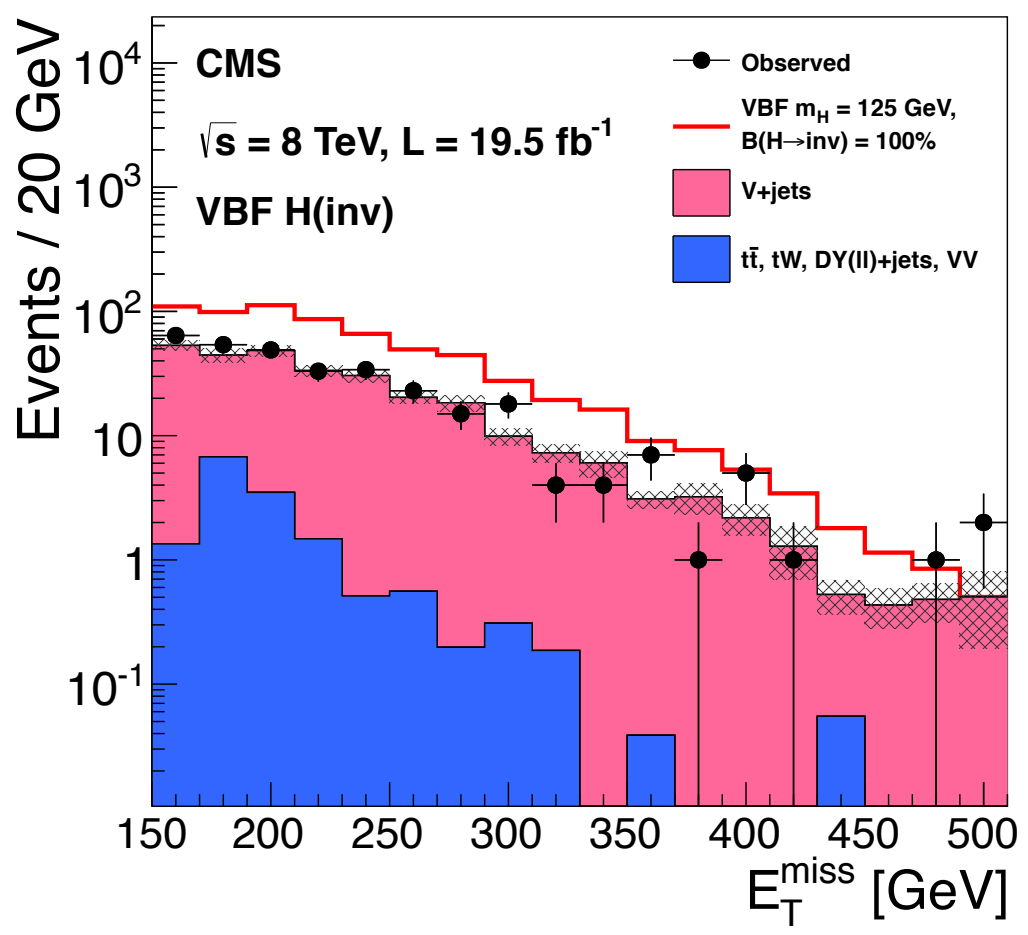

FIG. 15: $\mathbb{E}_{\mathrm{T}}$ distribution after full selection of the VBF analysis. [77]

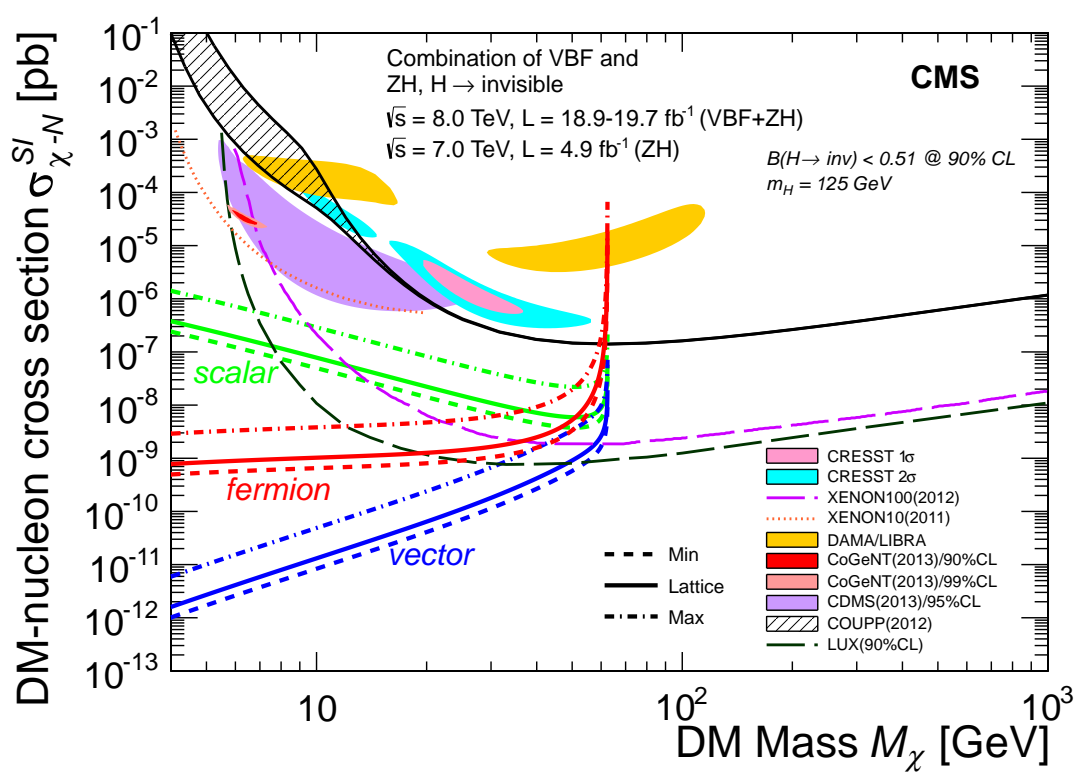

FIG. 16: Upper limits at 90\% CL on the WIMP-nucleon cross section as a function of the DM mass, derived from the experimental upper limit on $\mathcal{B} \mathcal{R}(H \rightarrow$ inv. $)$ for $m_{H}=125 \mathrm{GeV}$, in the scenarios where the DM candidate is a scalar, a vector, or a Majorana fermion. [78] 


\section{Limits derived from the total Higgs Branching Ratio}

The ATLAS collaboration [79] constrains invisible Higgs decays also using the combined measurements of $H \rightarrow \gamma \gamma, H \rightarrow Z Z \rightarrow 4 \ell, H \rightarrow W W \rightarrow \ell \nu \ell \nu, H \rightarrow \tau \tau$, and $H \rightarrow b \bar{b}$

in combination with the upper limit on $Z H \rightarrow \ell \ell+\mathbb{E}_{\mathrm{T}}$ to derive an upper limit on the branching ratio to invisible states. Standard Model values for the couplings of the Higgs boson to massive particles are assumed for Higgs boson production and decay. Effective couplings to photons and gluons, $\kappa_{\gamma}, \kappa_{g}$ respectively, are assumed to absorb potential loop contributions of new particles. The ratio of total width of the Higgs boson to Standard Model expectation is parametrized by $\kappa_{H}^{2}$ :

$$
\begin{aligned}
\kappa_{H}^{2} & =\Gamma_{H} / \Gamma_{H, \mathrm{SM}}=\sum_{i} \kappa_{i}^{2} /\left(1-\mathcal{B R}_{i}\right) \\
\sum_{i} \kappa_{i}^{2} & =0.0023 \kappa_{\gamma}^{2}+0.085 \kappa_{g}^{2}+0.91
\end{aligned}
$$

and a likelihood scan is performed. With constraints applied to ensure that the fit produces physical values the best fit yields $B R_{i}<0.37$ (0.39) observed (expected) at 95\% CL using the combination of all channels. Under the assumptions $2 \cdot m_{\chi} \leq m_{H}$ and the resulting Higgs boson decays to WIMP pairs account entirely for $B R_{i}$ limits are derived on the Higgs coupling to mass depending on the WIMP mass and parametrized as limit on the direct WIMP-nucleon scattering cross section via Higgs exchange. Figure 17 presents the limit on the spin-independent WIMP-nucleon scattering cross section.

The upper limits from ZH searches can be translated to WIMP-nucleon cross section. The current upper bound from these limits are similar for either collaboration, while the ATLAS limits perform slightly better. Comparison of these upper limits is shown in Figure 18 for scalar, vector, Majorana fermion DM candidates. Although for $m_{\chi}>m_{H} / 2$ the limits obtained by LUX are stronger compared to collider searches whereas at lower masses bounds from $Z H$ dominated for spin-independent interactions.

\section{CURRENT BOUNDS}

Figure 19 shows the current $90 \%$ CL lower bounds on suppression scale $\Lambda$ as a function of $M_{\chi}$ for various DM operators considered in different collider searches. These DM operators 


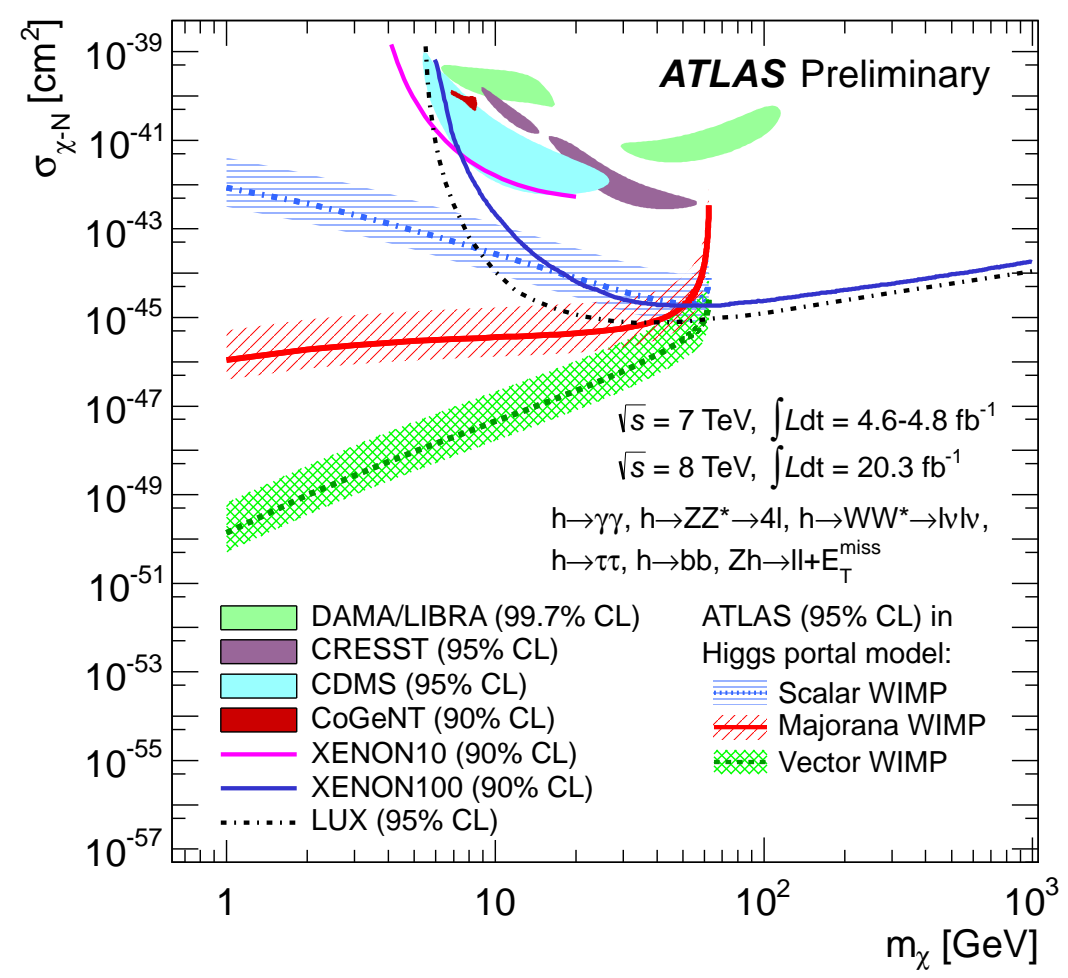

FIG. 17: ATLAS upper limit at 95\% CL on the WIMP-nucleon scattering cross section in a Higgs portal model as a function of the mass of the dark matter particle, shown separately for a scalar, Majorana fermion, or vector boson WIMP. Excluded and allowed regions from direct detection experiments are also shown. These are spin-independent results obtained directly from searches for nuclei recoils from elastic scattering of WIMPs, rather than being inferred indirectly through Higgs boson exchange in the Higgs portal model.

include C1, D1, D5, D8, D9 and D11. The ATLAS results from mono- $W / Z(j j)+\chi \chi$ gives the strongest lower limits for C1, D1, D5, and D9 DM operators, reflecting the impressive control of systematics possible in boosted boson searches. The best bounds for the D11 operator comes from the mono-jet search done by the CMS collaboration (as expected for an operator coupling only to gluons), while strongest bound for D8 are observed in mono$W(\ell \nu)+\chi \chi$, as it was not included in the hadronic decay search of ATLAS. For D5 and D8 DM operators results are presented for different coupling scenarios that determine the type of interference as discussed in Sec III.

The current status of WIMP-nucleon cross section upper limit from collider searches is presented in Figure 20 for different DM operators. The best bounds for each type of operator 


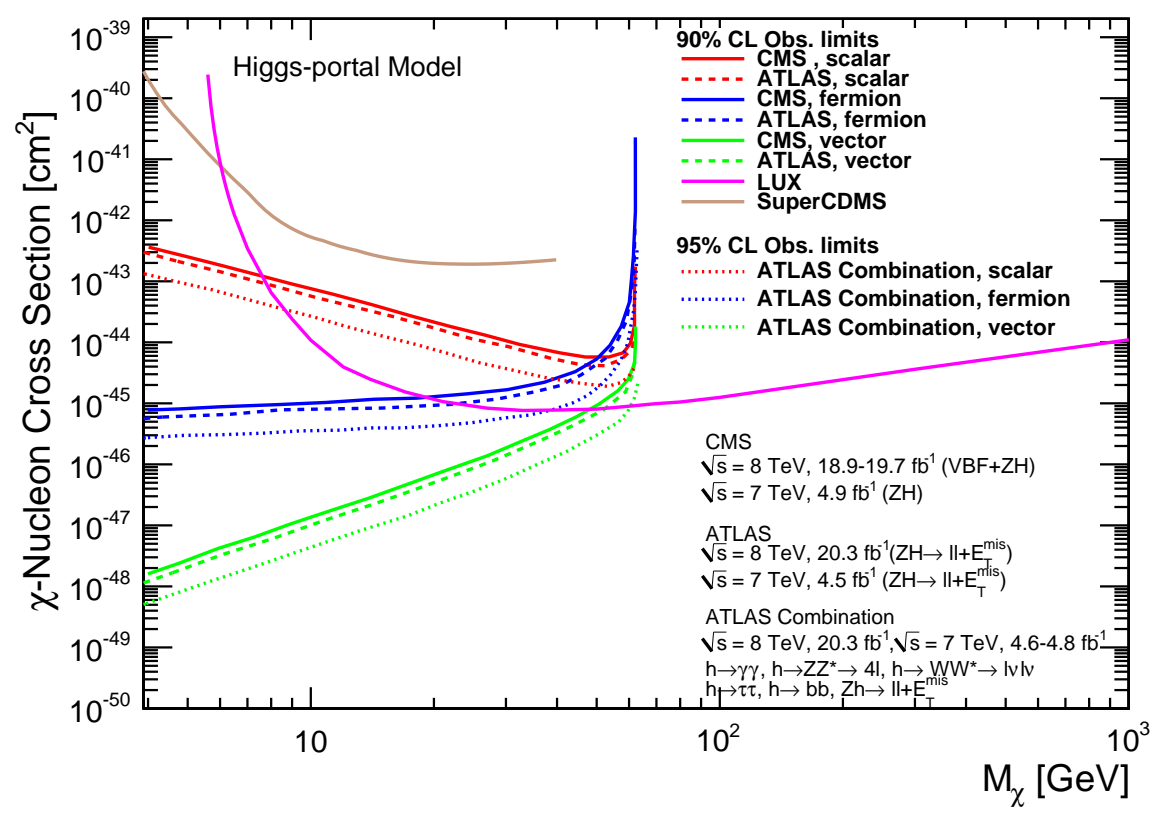

FIG. 18: Observed 90\% CL upper limit for WIMP-nucleon cross section with scalar, vector and Majorana DM particle from $Z(\rightarrow \ell \ell) H$ final state. These results from the CMS and the ATLAS collaboration are also compared with latest direct detection bound from LUX [58, SuperCDMS 60.

are from the similar final state as in case of $\Lambda$. For D8 operator the best upper limits are from the CMS collaboration in mono-jet final state.

The different searches summarized here have not found any evidence of DM production. The WIMP-nucleon cross section limits from collider are comparable to direct searches. Present lower bounds on the scale $(\Lambda)$ of these interactions vary from few $\mathrm{GeV}$ (for $\mathrm{C} 1$ ) to few TeVs (for D9) for different type of DM operators and couplings discussed in this article.

\section{Acknowledgements}

The research of WS is partly supported by the US Department of Energy under contract DE-FG02-04ER41268. SC and MT are supported by the grant DE-SC000999. AA is 


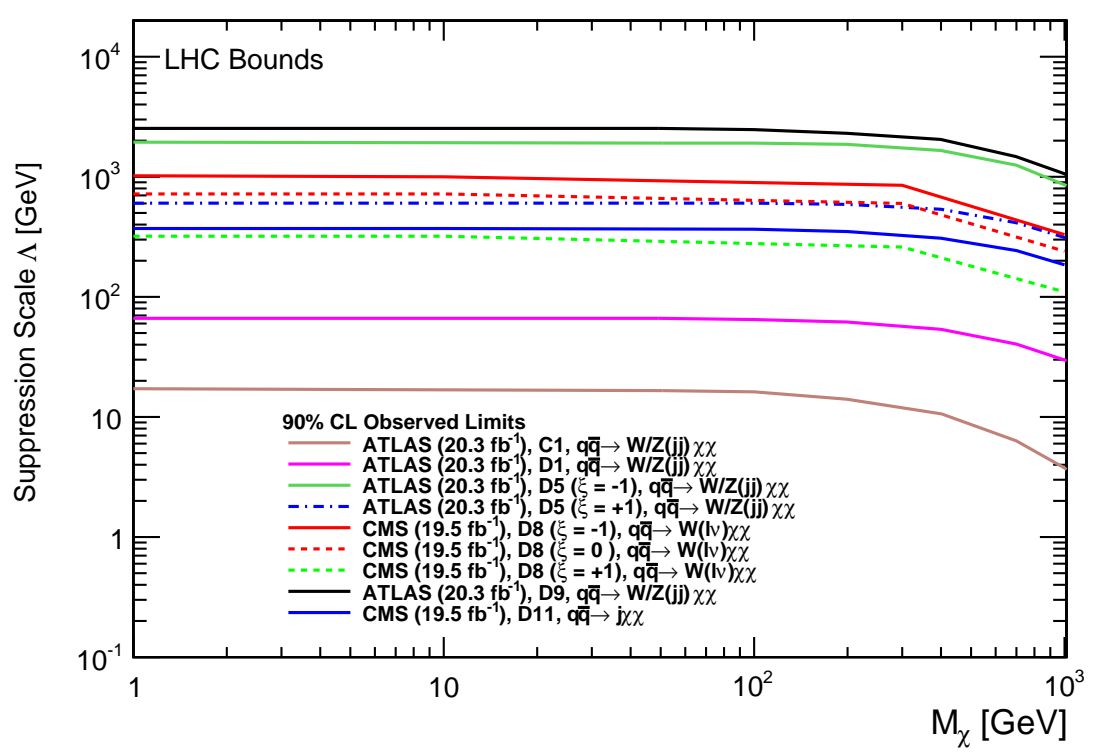

FIG. 19: The current bounds for different DM operators from LHC searches with different final state.

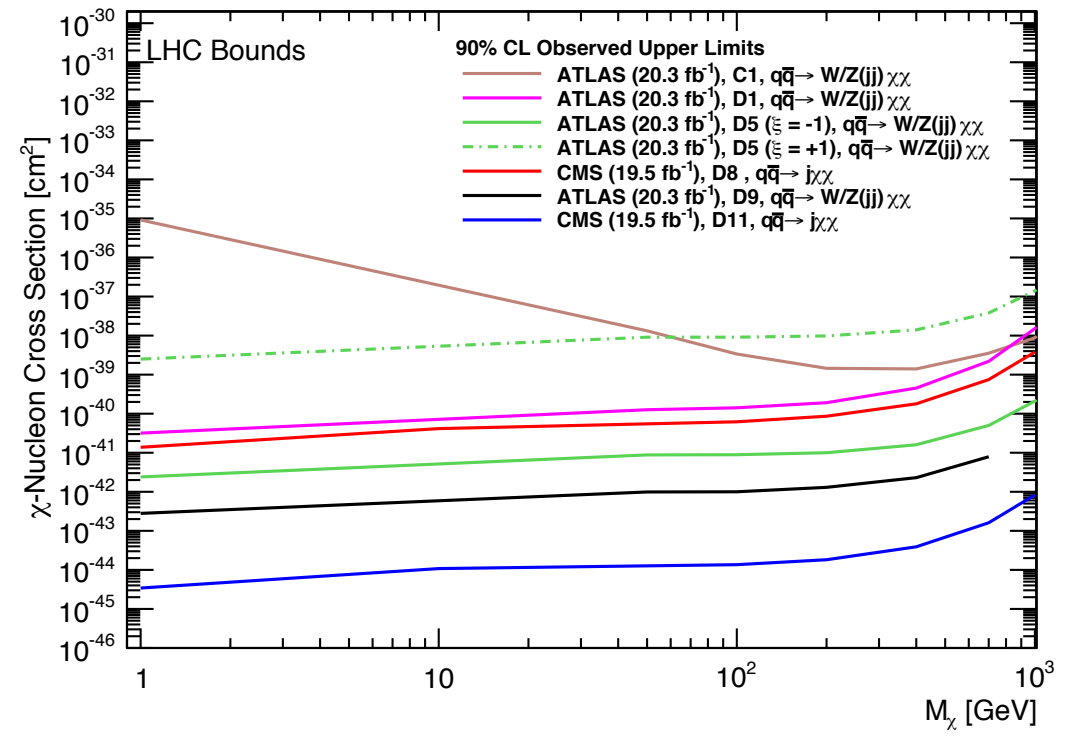

FIG. 20: The current bounds on upper limit for $\chi$-nucleon cross section for different DM operators from collider searches with different final state. 
supported by the US Department of Energy under contract DE-FG02-13ER41942.

[1] G. Aad et al. (ATLAS Collaboration), Phys.Lett. B716, 1 (2012), 1207.7214.

[2] S. Chatrchyan et al. (CMS Collaboration), Phys.Lett. B716, 30 (2012), 1207.7235.

[3] L. Bergstrom, Annalen Phys. 524, 479 (2012), 1205.4882.

[4] P. Ade et al. (Planck Collaboration) (2013), 1303.5076.

[5] M. S. Madhavacheril, N. Sehgal, and T. R. Slatyer (2013), 1310.3815.

[6] J. L. Feng and J. Kumar, Phys.Rev.Lett. 101, 231301 (2008), 0803.4196.

[7] L. Goodenough and D. Hooper (2009), 0910.2998.

[8] D. Hooper and L. Goodenough, Phys.Lett. B697, 412 (2011), 1010.2752.

[9] D. Hooper and T. Linden, Phys.Rev. D84, 123005 (2011), 1110.0006.

[10] K. N. Abazajian and M. Kaplinghat, Phys.Rev. D86, 083511 (2012), 1207.6047.

[11] M. Su and D. P. Finkbeiner (2012), 1206.1616.

[12] T. Daylan, D. P. Finkbeiner, D. Hooper, T. Linden, S. K. N. Portillo, et al. (2014), 1402.6703.

[13] M. Ackermann et al. (Fermi-LAT Collaboration), Phys.Rev. D89, 042001 (2014), 1310.0828.

[14] V. A. Mitsou, Int.J.Mod.Phys. A28, 1330052 (2013), 1310.1072.

[15] J. Goodman, M. Ibe, A. Rajaraman, W. Shepherd, T. M. Tait, et al., Phys.Lett. B695, 185 (2011), 1005.1286 .

[16] J. Goodman, M. Ibe, A. Rajaraman, W. Shepherd, T. M. Tait, et al., Nucl.Phys. B844, 55 (2011), 1009.0008.

[17] P. J. Fox, R. Harnik, J. Kopp, and Y. Tsai, Phys.Rev. D84, 014028 (2011), 1103.0240.

[18] A. Rajaraman, W. Shepherd, T. M. Tait, and A. M. Wijangco, Phys.Rev. D84, 095013 (2011), 1108.1196.

[19] P. J. Fox, R. Harnik, J. Kopp, and Y. Tsai, Phys.Rev. D85, 056011 (2012), 1109.4398.

[20] A. Friedland, M. L. Graesser, I. M. Shoemaker, and L. Vecchi, Phys.Lett. B714, 267 (2012), 1111.5331.

[21] K. Cheung, P.-Y. Tseng, Y.-L. S. Tsai, and T.-C. Yuan, JCAP 1205, 001 (2012), 1201.3402.

[22] T. Aaltonen et al. (CDF Collaboration), Phys.Rev.Lett. 108, 211804 (2012), 1203.0742.

[23] P. J. Fox, R. Harnik, R. Primulando, and C.-T. Yu, Phys.Rev. D86, 015010 (2012), 1203.1662.

[24] Y. Bai and T. M. Tait, Phys.Lett. B723, 384 (2013), 1208.4361. 
[25] Y. J. Chae and M. Perelstein, JHEP 1305, 138 (2013), 1211.4008.

[26] P. J. Fox and C. Williams, Phys.Rev. D87, 054030 (2013), 1211.6390.

[27] L. M. Carpenter, A. Nelson, C. Shimmin, T. M. Tait, and D. Whiteson, Phys.Rev. D87, 074005 (2013), 1212.3352 .

[28] N. Zhou, D. Berge, and D. Whiteson, Phys.Rev. D87, 095013 (2013), 1302.3619.

[29] J. M. Cornell, S. Profumo, and W. Shepherd, Phys.Rev. D88, 015027 (2013), 1305.4676.

[30] R. C. Cotta, A. Rajaraman, T. M. P. Tait, and A. M. Wijangco (2013), 1305.6609.

[31] M. R. Buckley, Phys.Rev. D88, 055028 (2013), 1308.4146.

[32] R. Essig, J. Mardon, M. Papucci, T. Volansky, and Y.-M. Zhong, JHEP 1311, 167 (2013), 1309.5084 .

[33] P. Agrawal and V. Rentala (2013), 1312.5325.

[34] A. Crivellin, F. D’Eramo, and M. Procura (2014), 1402.1173.

[35] G. Busoni, A. De Simone, J. Gramling, E. Morgante, and A. Riotto (2014), 1402.1275.

[36] M. Song, G. Li, W.-G. Ma, R.-Y. Zhang, and J.-Y. Guo (2014), 1403.2142.

[37] S. Davidson (2014), 1403.5161.

[38] N. Lopez, L. M. Carpenter, R. Cotta, M. Frate, N. Zhou, et al. (2014), 1403.6734.

[39] G. Artoni, T. Lin, B. Penning, G. Sciolla, and A. Venturini (2013), 1307.7834.

[40] J. Goodman, M. Ibe, A. Rajaraman, W. Shepherd, T. M. Tait, et al., Phys.Rev. D82, 116010 (2010), 1008.1783.

[41] H. Goldberg and L. J. Hall, Phys.Lett. B174, 151 (1986).

[42] A. Buras, P. Gambino, M. Gorbahn, S. Jager, and L. Silvestrini, Phys.Lett. B500, 161 (2001), hep-ph/0007085.

[43] H. M. Georgi, S. L. Glashow, M. E. Machacek, and D. V. Nanopoulos, Phys. Rev. Lett. 40, 692 (1978), URL http://link.aps.org/doi/10.1103/PhysRevLett.40.692.

[44] U. Haisch, F. Kahlhoefer, and J. Unwin, JHEP 1307, 125 (2013), 1208.4605.

[45] I. M. Shoemaker and L. Vecchi, Phys.Rev. D86, 015023 (2012), 1112.5457.

[46] G. Busoni, A. De Simone, E. Morgante, and A. Riotto, Phys.Lett. B728, 412 (2014), 1307.2253.

[47] H. An, L.-T. Wang, and H. Zhang (2013), 1308.0592.

[48] A. DiFranzo, K. I. Nagao, A. Rajaraman, and T. M. P. Tait, JHEP 1311, 014 (2013), 1308.2679. 
[49] O. Buchmueller, M. J. Dolan, and C. McCabe, JHEP 1401, 025 (2014), 1308.6799.

[50] S. Chang, R. Edezhath, J. Hutchinson, and M. Luty, Phys.Rev. D89, 015011 (2014), 1307.8120.

[51] Tech. Rep. CMS-PAS-EXO-12-048, CERN, Geneva (2013).

[52] D. Alves et al. (LHC New Physics Working Group), J.Phys. G39, 105005 (2012), 1105.2838.

[53] J. Goodman and W. Shepherd (2011), 1111.2359.

[54] S. Profumo, W. Shepherd, and T. Tait (2013), 1307.6277.

[55] N. Arkani-Hamed, A. Delgado, and G. Giudice, Nucl.Phys. B741, 108 (2006), hep$\mathrm{ph} / 0601041$.

[56] R. Cotta, J. Hewett, M. Le, and T. Rizzo, Phys.Rev. D88, 116009 (2013), 1210.0525.

[57] J.-Y. Chen, E. W. Kolb, and L.-T. Wang, Phys.Dark Univ. 2, 200 (2013), 1305.0021.

[58] D. Akerib et al. (LUX Collaboration) (2013), 1310.8214.

[59] M. Felizardo, T. Girard, T. Morlat, A. Fernandes, A. Ramos, et al., Phys.Rev.Lett. 108, 201302 (2012), 1106.3014.

[60] R. Agnese et al. (SuperCDMS Collaboration) (2014), 1402.7137.

[61] M. Aartsen et al. (IceCube collaboration), Phys.Rev.Lett. 110, 131302 (2013), 1212.4097.

[62] S. Archambault et al. (PICASSO Collaboration), Phys.Lett. B711, 153 (2012), 1202.1240.

[63] E. Aprile et al. (XENON100 Collaboration), Phys.Rev.Lett. 111, 021301 (2013), 1301.6620.

[64] G. Aad et al. (ATLAS Collaboration), Phys.Rev.Lett. 110, 011802 (2013), 1209.4625.

[65] S. Chatrchyan et al. (CMS Collaboration), Phys.Rev.Lett. 108, 261803 (2012), 1204.0821.

[66] Tech. Rep. CMS-PAS-EXO-12-047, CERN, Geneva (2014).

[67] G. Aad et al. (ATLAS Collaboration), JHEP 1304, 075 (2013), 1210.4491.

[68] Tech. Rep. ATLAS-CONF-2012-147, CERN, Geneva (2012).

[69] S. Chatrchyan et al. (CMS Collaboration), JHEP 1209, 094 (2012), 1206.5663.

[70] M. Beltran, D. Hooper, E. W. Kolb, Z. A. Krusberg, and T. M. Tait, JHEP 1009, 037 (2010), 1002.4137.

[71] M. Ackermann et al. (Fermi-LAT collaboration), Phys.Rev.Lett. 107, 241302 (2011), 1108.3546 .

[72] E. Komatsu et al. (WMAP Collaboration), Astrophys.J.Suppl. 192, 18 (2011), 1001.4538.

[73] G. Aad et al. (ATLAS Collaboration), Phys.Rev.Lett. 112, 041802 (2014), 1309.4017.

[74] Y. L. Dokshitzer, G. Leder, S. Moretti, and B. Webber, JHEP 9708, 001 (1997), hep- 
$\mathrm{ph} / 9707323$.

[75] Tech. Rep. CMS-PAS-EXO-13-004, CERN, Geneva (2013).

[76] G. Aad et al. (ATLAS Collaboration) (2014), 1404.0051.

[77] G. Aad et al. (ATLAS Collaboration) (2014), 1402.3244.

[78] S. Chatrchyan et al. (CMS Collaboration) (2014), 1404.1344.

[79] Tech. Rep. ATLAS-CONF-2014-010, CERN, Geneva (2014). 